\title{
Laser Fluorescence Spectroscopy: Application in Determining the Individual Photophysical Parameters of Proteins
}

\author{
Alexander A. Banishev ${ }^{*}$ \\ Department of Physics and Astronomy, University of California, Riverside, CA, \\ USA
}

\section{Introduction}

This work was initiated by the problem of investigating the photophysical properties of complex protein molecules and performing the diagnostics of such molecules in water environment. At the present time, fluorescence spectroscopy (fluorimetry) is widely used to study complex organic compounds (COC) (Lakowicz, 1999). Together with spectrophotometry these methods form the basis for fast and nondestructive diagnostics of COC in the natural environment, i.e. they present the diagnostic methods in vivo and in situ. However, the conventional (linear) fluorescence spectroscopy methods can not provide complete information on fluorescent objects under study because of insufficient selectivity (fluorescence bands of most COC are broad and structureless at room temperature).

The capabilities of fluorescence spectroscopy can be enhanced by using the methods of laser fluorimetry, in particular nonlinear laser fluorimetry (Fadeev et al., 1999). This method allows one to get information on the molecular level and determine the photophysical parameters of molecules (absorption cross section, lifetime in excitation state, intersystem crossing and energy transfer rates, etc.). Furthermore, the parameters can be measured in vivo and in situ in the absence of a priori information, which is necessary for conventional spectroscopic methods (for example, molecular concentration (Banishev et al., 2009)).

The diagnostics of protein complexes is an intricate problem if a molecule contains more than one absorption/fluorescent center (Permyakov, 1992). The problem becomes much more complex if, in addition, the protein specimen (ensemble of molecules) is a mixture of several chemically nonidentical types of molecules (subensembles) which cannot be separated, i.e. their partial concentrations are unknown. The second situation is typical for the special kind of proteins, namely, fluorescent proteins (FPs) (Piatkevich et al., 2010). The solutions of FPs are usually mixtures of several types of molecules, which are chemically different and have their own set of photophysical properties (Verkhusha et al., 2004). In this case for unambiguous interpretation of experimental data it is necessary to make simultaneous measurements of a large number of parameters, i.e. to simultaneously apply (or, better, synthesize) several spectroscopic methods.

${ }^{*}$ Corresponding Author 
In this chapter, a new approach based on the simultaneous use of nonlinear laser fluorimetry, spectrophotometry and conventional fluorimetry methods is presented. The approach allows us to in vivo determine the individual photophysical parameters of fluorophores in multi-fluorophore protein complexes. The approach has been applied for investigation of the photophysical properties of the protein molecules of different complexity. Two classes of proteins have been chosen, namely, serum albumins (by the examples of human and bovine serum albumins) and fluorescent proteins (by the example of monomeric red FP mRFP1). The following new results are presented.

i. The photophysical parameters such as (a) true absorption cross section (at $266 \mathrm{~nm}$ ) of tryptophan and intersystem crossing rate in single-tryptophan-containing protein human serum albumin and (b) true absorption cross section (at $266 \mathrm{~nm}$ ) of tryptophans and rate of energy transfer between them in two-tryptophan-containing protein bovine serum albumin have been determined.

ii. The complete solution of the task of determining the photophysical parameters of all mRFP1 spectral forms is given. The mechanism of photophysical processes in the spectral forms under their excitation by UV radiation (at $266 \mathrm{~nm}$ ) has been clarified.

iii. The study of the influence of a single amino acid substitution in mRFP1 protein on individual photophysical parameters of the chromophore (a heterogroup ${ }^{1}$ responsible for light absorption and fluorescence in the visible wavelength range) of fluorescent spectral form is performed. The 66th amino acid residue (glutamine 66) has been chosen as a position to be replaced. This residue participates in formation of the chromophore and, as was shown in (Banishev et al., 2009), its substitution by polar serine or cysteine changes the spectral and photophysical properties of the resultant mutant of the mRFP1. In the present work this study has been extended. The optical properties of new variants of mRFP1 with polar (asparagine, histidine) and non-polar (alanine, leucine, phenylalanine) substitution have been investigated. It was found that the individual extinction coefficient of the chromophore and the position of the steady-state spectra of the proteins with polar substitution correlate with the volume of the substituted amino acid residue at position 66. The explanation of this effect is given.

Except for this key target, the methodological task has been put, namely, to demonstrate the unique capabilities of the nonlinear laser fluorimetry method (which is not, so far, well known in a wide circle of opticians) on the specific object.

\section{The method of nonlinear laser fluorescence spectroscopy}

The fluorescence signal from fluorophores of complex organic compound (COC) under powerful laser excitation is represented as the nonlinear function of the number of detected fluorescence photons $N_{F l}$ (or fluorescence intensity $I_{F l}$ ) on the photon fluxes $F$ of pumping radiation (Filipova et al., 2001). The dependence $N_{F l}(F)$ is called fluorescence saturation curve, its typical view is represented in the Fig. 1(a). There are several reasons for that nonlinear dependence: the non-zero lifetime of organic molecules in excited state; intercombination conversion; intermolecular interactions including singlet-singlet annihilation, etc.

${ }^{1}$ The heterogroup is called a chromophore, even when it produces fluorescence, in other words, it is a fluorophore. 

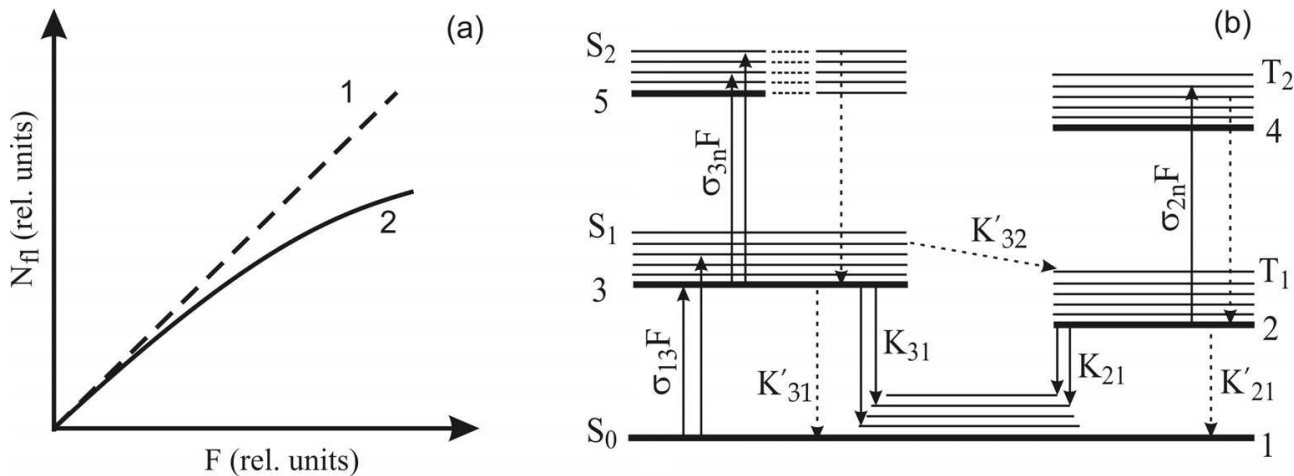

Fig. 1. (a) The $N_{F l}(F)$ dependencies (see text): (1) in the absence of fluorescence saturation, and (1b) when saturation appears (for most COC at $F>10^{23} \mathrm{~cm}^{-2} \mathrm{~s}^{-1}$ (Fadeev et al., 1999)).

(b) Photophysical processes in COC (Lakowicz, 1999), without accounting for intermolecular interactions. Solid and dotted vertical lines are radiation and radiationless transitions respectively, $S_{i}$ are singlet states and $T_{i}$ are triplet states.

The parameters of saturation curves depend on photophysical characteristics of molecules fluorophores, so that such characteristics can be extracted from these curves after resolving an inverse problem (Fadeev et al., 1999). This is a basement of nonlinear laser fluorimetry as a method for investigation of photophysical properties of COC. To solve the inverse problem, we should first calculate (either analytically or numerically) the theoretical saturation curves by using the fluorescence response formation model of an ensemble of fluorescent molecules under their excitation by laser radiation. In present work two models have been used: the conventional model of fluorescence response formation and the model of localized donor-acceptor (LDA) pairs.

The conventional model (Banishev et al., 2008a, 2009) in describing the fluorescence response is represented as a system of equations that describes the kinetics of concentration of COC molecules at the corresponding energy states (Fig. 1(b)). In the case of monomolecular solutions of non-interactive organic compounds, we must give priority to the following photophysical parameters when defining the saturation curve:

- the absorption cross section $\sigma \equiv \sigma_{13}$, which determines the probability of a molecule transition from the ground singlet state $S_{0}$ (level 1) to the first excited state $S_{1}$ (level 3) stimulated by a photon flux with the density $F$;

- the full lifetime $\tau \equiv \tau_{3}$ of the molecule in the $S_{1}$ state (the fluorescence decay time);

- the quantum yield $\eta_{T}=K_{32}^{\prime} / K_{3}$ of the molecule transition to the lower triplet state $\mathrm{T}_{1}$ (level 2) due to the intersystem crossing, where $K_{3}=\tau-1=K_{31}+K_{31}^{\prime}+K^{\prime}{ }_{32} ; K_{31}$ and $K_{31}^{\prime}$ are the rates of radiative and radiationless transitions from $S_{1}$ to $S_{0} ; K_{32}^{\prime}$ is the rate of the transition from $S_{1}$ to $T_{1}$.

The model of the fluorescence response, which takes into account processes pointed out above, can be described by the following set of kinetic equations (Fadeev et al., 1999): 


$$
\begin{aligned}
& \frac{\partial n_{1}(t, \vec{r})}{\partial t}=-F(t, \vec{r}) \cdot \sigma \cdot\left[n_{0}(t, \vec{r})-n_{3}(t, \vec{r})-n_{2}(t, \vec{r})\right]+\left(K_{3}-K_{32}^{\prime}\right) \cdot n_{3}(t, \vec{r}) \\
& \frac{\partial n_{3}(t, \vec{r})}{\partial t}=F(t, \vec{r}) \cdot \sigma \cdot\left[n_{0}(t, \vec{r})-n_{3}(t, \vec{r})-n_{2}(t, \vec{r})\right]-K_{3} \cdot n_{3}(t, \vec{r}) \\
& \frac{\partial n_{2}(t, \vec{r})}{\partial t}=K_{32}^{\prime} \cdot n_{3}(t, \vec{r}) \\
& n_{0}=n_{1}+n_{2}+n_{3},
\end{aligned}
$$

where $n_{0}$ is the total concentration of molecules; $n_{3}, n_{2}$, and $n_{1}$ are concentrations of molecules in the $S_{1}, T_{1}$ and $S_{0}$ states, respectively; $F(t, \vec{r})$ is the photon flux density of exciting radiation at the coordinate point $\vec{r}$ at instant of time $t$. The rest of parameters are defined above. In model (1a), the transition from $T_{1}$ to $S_{0}$ is neglected. This assumption is valid if the light pulse duration $\left(t_{p}\right)$ is much less than the lifetime in the $\mathrm{T}_{1}$ state, i.e. $t_{p}$ is much less then $\left(K_{21}+K_{21}^{\prime}\right)^{-1}$. For pulse lasers often used in laser fluorescence spectroscopy, the $t_{p}$ is $\sim 10 \mathrm{~ns}$, and this condition is fulfilled.

The conventional model (1a) describes the processes in a system in the absence of interaction between molecules. If there is the interaction and an ensemble of fluorophores generating the fluorescence response consists of subensembles of the donor and acceptor molecules, then the conventional approach is reduced to two systems of kinetic equations, i.e. separately for each subensemble. The term describing the energy transfer is in this case a "cross term" that connects these two systems of equations. Such model, based on separate mathematical description of two subensembles, is able to describe the fluorescence response when each molecule of the donor is surrounded by a large number of the acceptor molecules onto which the energy transfer can occur (Agranovich \& Galanin, 1982) (i.e. the possibility that the donor molecule and all the locally surrounding it acceptor molecules simultaneously stay in the excitation state is excludes). The situation like this is typical for a concentrated binary solution of single-fluorophore molecules (for example, dye solutions with the concentration higher than $10^{-4} \mathrm{M}$ ) or for complexes with high local concentration (Fadeev et al., 1999), such as phytoplankton. The energy transfer process in that case is called the intermolecular one.

If there is a donor-acceptor pair within a single molecule (i.e. we have a molecule with a LDA pair), the situation is possible when the donor and the acceptor are simultaneously in an excited state. Therefore, the description of the energy transfer in the framework of a conventional scheme is impossible and the model (1a) should be modified. Let us note that the molecular objects with LDA pair are finding more and more wide applications at present time. Commonly, systems of this kind are constructed artificially from pairs of organic compounds, for example, from dye molecules (Srinivas et al., 2001) or FP macromolecules (Truong \& Ikura , 2001). In (Banishev et al., 2008b) a fluorescence response formation model of an ensemble of LDA pairs has been suggested by the author. The model makes it possible to describe the energy transfer inside a LDA pair, disregarding the energy transfer between the pairs. The main idea of this approach consists in the following. Let us introduce a notion of the collective states of a LDA pair (Fig. 2); each of these states simultaneously describes both the donor state and the acceptor state: 


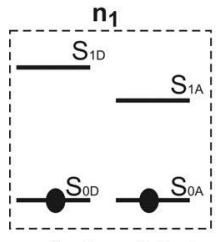

collective state 1

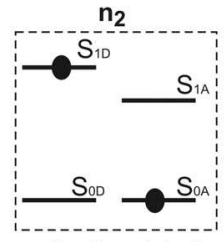

collective state 2

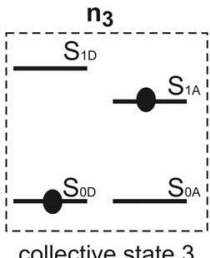

collective state 3

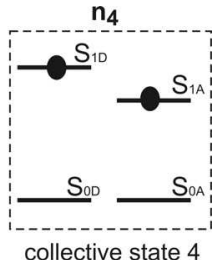

Fig. 2. The collective states of the LDA pair (nonmetering the process of singlet-singlet annihilation and intersystem crossing). The $S_{0 D}, S_{1 D}$ and $S_{0 A}, S_{1 A}$ are the energy levels of the donor and acceptor respectively.

- Collective state 1: the donor and the acceptor are in the ground state $\mathrm{S}_{0 \mathrm{D}}$ and $\mathrm{S}_{0 \mathrm{~A}}$, respectively; the concentration of such molecules is denoted as $n_{1} \equiv n_{1}(t, \vec{r})$.

- Collective state 2: the donor is in the first excited singlet state $S_{1 \mathrm{D}}$, and the acceptor is in the state $S_{0 A}$; the concentration of such molecules is denoted as $n_{2} \equiv n_{2}(t, \vec{r})$.

- Collective state 3: the donor is in the state $S_{0 D}$, and the acceptor is in the first excited singlet state $S_{1 A}$; the concentration of such molecules is denoted as $n_{3} \equiv n_{3}(t, \vec{r})$.

- Collective state 4: the donor and the acceptor are in the first excited singlet state $S_{1 D}$ and $\mathrm{S}_{1 \mathrm{~A}}$, respectively; the concentration of such molecules is denoted as $n_{4} \equiv n_{4}(t, \vec{r})$.

As a result, there is no need to describe the fluorescence response from a sub-ensemble of donor and acceptor molecules separately, i.e. it is unnecessary to create two systems of equations (one for a donor sub-ensemble and one for an acceptor sub-ensemble) similar to (1a), as it takes place in the conventional approach. Instead of this, the system of equations that describes the populations of the collective states can be written. The dynamics of variation in the concentrations of these four collective states of the LDA pair (nonmetering the process of intersystem crossing in molecules of the donor and acceptor) is mathematically described by the following system of kinetic equations:

$$
\begin{aligned}
& \frac{\partial n_{1}}{\partial t}=-F(t, \vec{r}) \cdot\left(\sigma_{D}+\sigma_{A}\right) \cdot n_{1}+\frac{n_{2}}{\tau_{D}}+\frac{n_{3}}{\tau_{A}} \\
& \frac{\partial n_{2}}{\partial t}=-F(t, \vec{r}) \cdot \sigma_{A} \cdot n_{2}-\frac{n_{2}}{\tau_{D}}-K_{D A} \cdot n_{2}+F(t, \vec{r}) \cdot \sigma_{D} \cdot n_{1}+\frac{n_{4}}{\tau_{A}} \\
& \frac{\partial n_{3}}{\partial t}=-F(t, \vec{r}) \cdot \sigma_{D} \cdot n_{3}-\frac{n_{3}}{\tau_{A}}+F(t, \vec{r}) \cdot \sigma_{A} \cdot n_{1}+\frac{n_{4}}{\tau_{D}}+K_{D A} \cdot n_{2}+K_{S S} \cdot n_{4} \\
& \frac{\partial n_{4}}{\partial t}=F(t, \vec{r}) \cdot \sigma_{A} \cdot n_{2}+F(t, \vec{r}) \cdot \sigma_{D} \cdot n_{3}-\frac{n_{4}}{\tau_{D}}-\frac{n_{4}}{\tau_{A}}-K_{S S} \cdot n_{4} \\
& n_{1}+n_{2}+n_{3}+n_{4}=n_{0},
\end{aligned}
$$

where $\tau_{D}, \tau_{A}$ and $\sigma_{D}, \sigma_{\mathrm{A}}$ are the lifetime and absorption cross section of the donor (denoted by $\mathrm{D}$ ) and acceptor (denoted by A) as it defined above; $K_{D A}$ is the rate of the energy transfer from the excited donor to the unexcited acceptor; $K_{S S}$ is the rate of energy transfer from the excited donor to the excited acceptor (singlet-singlet annihilation (Fadeev et al., 1999); $F(t, \vec{r})$ is the photon flux density (see Eqs. (1a)); and $n_{0}$ is the total concentration of molecules containing a LDA pair. In this model, the following photophysical parameters are 
presented: the absorption cross section and the excited state lifetime of the donor and acceptor, the energy transfer rates $K_{D A}$ and $K_{S S}$.

By solving systems (1a) and (1b) numerically, one can find the concentration of the fluorescent molecules in the excited state and calculate the number of fluorescence photons $N_{F l}$, emitted from the volume $V$ after the action of the laser pulse (Filipova et al., 2001; Fadeev et al., 1999). The theoretical saturation curve for the model (1a) can be calculated from following equation:

$$
N_{F l}(\lambda)=K_{31} \cdot \int_{V} d \vec{r} \int_{-\infty}^{+\infty} n_{3}(t, \vec{r}) d t
$$

For the model (1b), for the donor (2b) and the acceptor (2c) curves, respectively:

$$
\begin{aligned}
& N_{F l}^{D}(\lambda)=\tau_{D}^{-1} \cdot \eta_{D} \cdot \int_{V} d \vec{r} \int_{-\infty}^{+\infty}\left(n_{2}(t, \vec{r})+n_{4}(t, \vec{r})\right) d t \\
& N_{F l}^{A}(\lambda)=\tau_{A}^{-1} \cdot \eta_{A} \cdot \int_{V} \overrightarrow{d r} \int_{-\infty}^{+\infty}\left(n_{3}(t, \vec{r})+n_{4}(t, \vec{r})\right) d t
\end{aligned}
$$

where $\eta_{D}$ and $\eta_{A}$ are the fluorescence quantum yield, which is defined as the ratio of the radiation decay rate of $S_{1}$ state to the sum of all rates of $S_{1}$ state decay (i.e. $\eta \equiv K_{31} / K_{3}$ ), of the donor and acceptor, correspondingly; $\lambda$ is the fluorescence registration wavelength. Other symbols are defined in Eqs. (1a).

In considered model (1a), the fluorescence saturation is caused by a finite lifetime $\tau$ and by intercombination conversion. In model $(1 b)$, due to the finite fluorescence lifetime and due to the saturation of the energy transfer channels. Let us note that the model $(1 \mathrm{~b})$ could be also supplemented with the intersystem crossing mechanisms, but preliminary experiments showed that at the given parameters of the laser radiation the process for albumins and mRFP1 is small compared to the mechanisms under study and contributes little to fluorescence saturation. Therefore, this mechanism has been excluded to increase the stability of the inverse problem solution (details and mathematical basement of inverse problem solution of nonlinear laser fluorimetry can be found elsewhere (Boychuk at al., 2000). For the same reason the induced processes from the excited states (two-photon absorption or photoizomerization, etc) have been excluded.

As was mention above, the photophysical parameters of fluorophores $\left(\sigma, K_{32}^{\prime}\right.$ and $\tau$ in the model (1a) and $\tau_{D}, \tau_{A}, \sigma_{D}, \sigma_{A}, K_{D A}$ and $K_{S S}$ in the model (1b)) can be determined from the dependence $N_{F l}(F)$, by solving the inverse problem. However, in experiments, it is convenient to normalize the number of detected fluorescence photons $N_{F l}$ to the reference signal (will denote as $N_{\text {Ref }}$ ), which can represent a part of exciting radiation directed to the reference channel of the detection system by a beamsplitter or a Raman scattering signal from water molecules (Fadeev et al., 1999). In this case, one has to deal with the dependence $[\Phi(F)]^{-1}=N_{\text {Ref }} / N_{F l}$ (which is also called a saturation curve, $\Phi(F)$ is the fluorescence parameter) rather than $N_{F l}(F)$. According to the practical experience such normalization also helps to increase the stability of the inverse problem solution. In the absence of saturation, $\Phi$ stops 
being dependent on photon flux density $F$ and tends to a constant which is denoted by $\Phi_{0}$ $\left(\left.\Phi_{0} \equiv \mathrm{im}\right|_{F \rightarrow 0}[\Phi(F)]\right)$. If in measurements of $\Phi_{0}$ the Raman scattering band of water molecules as a reference signal is used, then it is possible to find the fluorescence quantum yield of a complex organic compound (Filipova et al., 2001).

For the reasons pointed out in (Banishev et al., 2008a), the same laser fluorimeter has been optimized for measuring the nanosecond fluorescence decay (the kinetic mode of the fluorimeter operation). The curve represents the dependence of the number $N_{F l}\left(t_{d e l}\right)$ of fluorescence photons in the detector gate (with wide $t_{g}$ ) on the gate delay time $t_{\text {del }}$ with respect to a laser pulse. For the model (1a) an expression for kinetic curve can be written as:

$$
N_{F l}\left(t_{d e l}\right)=K_{31} \cdot \int_{V} d \vec{r} \int_{-t_{g} / 2+t_{d e l}}^{t_{g} / 2+t_{d e l}} n_{3}(t, \vec{r}) d t
$$

The $t_{d e l}$ changes discretely and proportionally to the detector gate step: $t_{d e l}=i \times t_{\text {step }}$, where $i$ is a number of the detector gate step. Similar expressions can be written for $(2 b, c)$. Gate position at which its centre coincided with the laser pulse maximum was taken as the zero delay $\left(t_{d e l}=0\right)$. This was detected by the maximum of water Raman line (Banishev et al., 2006).

By solving the inverse problem, the fluorescence lifetime $\tau$ of a fluorophore can be determined independently from the dependence $N_{F I}\left(t_{d e l}\right)$. In the experiment the fluorescence signal is measured in relative units. For comparison of the experimental data with the theoretical ones it is necessary to normalize the obtained experimental curve to the fluorescence intensity at some fixed time delay. This procedure, the fluorimeter capabilities in the kinetic mode and the corresponding theory can be found elsewhere (Banishev et al., 2006). The difference of such variant of kinetic fluorimetry from the conventional timeresolved fluorimetry is that the fluorescence is excited by a pulse with rather long duration ( $10 \mathrm{~ns}$ ), and for fluorescence registration an optical gated multichannel analyser is used. Whereas, the conventional time-resolved fluorimetry (Lakowicz, 1999) is based on the analysis of fluorescence decay curves after the excitation pulse, whose duration is much shorter than the lifetime of a fluorophore in the excitation state (picoseconds).

For determination of the photophysical parameters from experimental curves (solution of the inverse problem) the variation algorithm (Banishev et al., 2008a) was used. It is based on the procedure of minimizing the functional of the residue between the experimental curves and curves calculated from models $(1 \mathrm{a})$ or $(1 \mathrm{~b})$ by varying the photophysical parameters (Boychuk at al., 2000).

It is necessary to point out two distinctive features of nonlinear laser fluorimetry: (i) as the method implies detection of fluorescence photons (see Eqs. (2)), the photophysical parameters derived from the saturation curve relate only to a fluorescent molecule of COC; (ii) information on the concentration of fluorescent molecules is not used in deriving the photophysical parameters from the saturation curve (Banishev et al., 2009; Filipova et al., 2001). Thus, the method allows one to determine individual photophysical parameters of a molecule in the case when the following complex situation takes place: (i) the sample under study is a multicomponent ensemble of molecules, the absorption bands of its subensembles overlapping (i.e. when the sample is excited, all the subensembles absorb light); (ii) the concentrations of molecules from the subensembles in the mixture are a priori unknown. 


\section{Experimental}

\subsection{Nanosecond laser fluorescence spectrometer}

A homemade laser fluorimeter (Fig. 3) has been built for the experiments. The fluorimeter consist of a laser source, optical elements for light conversion, a fiber-optic cable, a cuvette and an optical multichannel analyser.

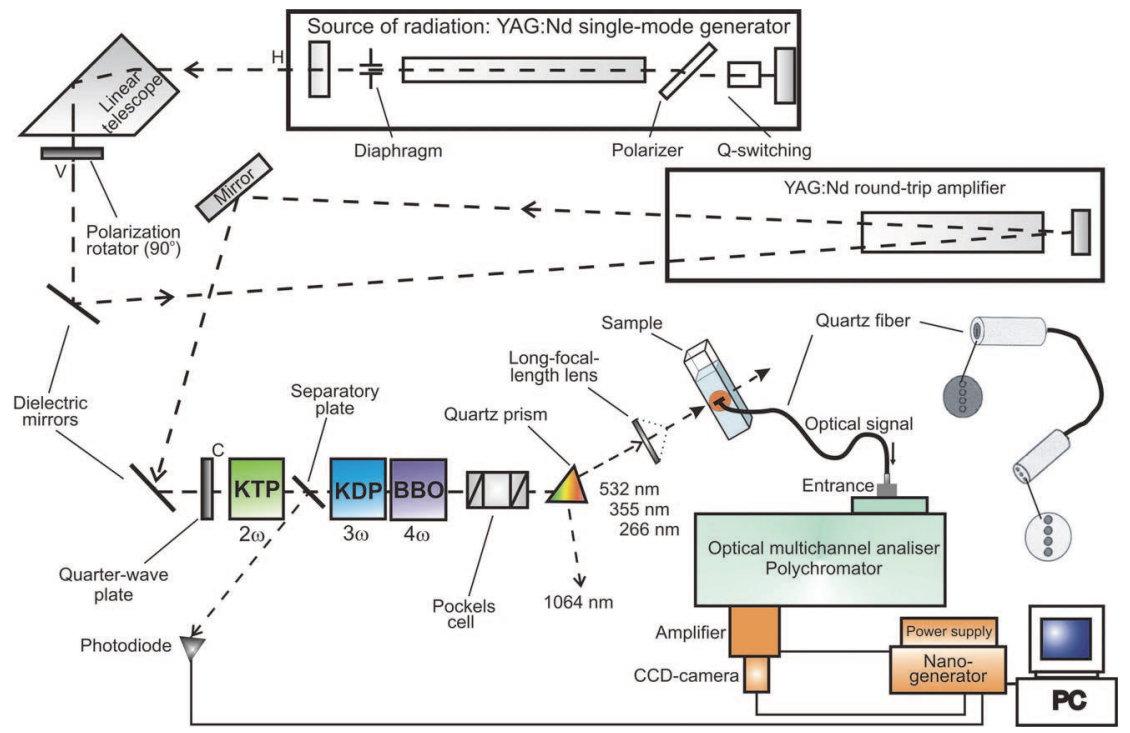

Fig. 3. Nanosecond laser fluorescence spectrometer scheme (for details see text). The letters $\mathrm{H}, \mathrm{V}$ and $\mathrm{C}$ denote the horizontal, vertical and circular light polarization.

\subsubsection{Laser excitation system}

The pulsed single mode Nd:YAG laser (the fundamental wavelength is $1064 \mathrm{~nm}$ ) with the set of nonlinear crystals for generating the $2 \mathrm{nd}(532 \mathrm{~nm})$, the $3 \mathrm{~d}(355 \mathrm{~nm})$ and the 4 th $(266$ $\mathrm{nm}$ ) harmonics of the fundamental radiation was used. At a pulse repetition frequency of 10 $\mathrm{Hz}$, the average pulse energy was: $5 \mathrm{~mJ}(532 \mathrm{~nm}), 2 \mathrm{~mJ}(355 \mathrm{~nm})$ and $0.7 \mathrm{~mJ}(266 \mathrm{~nm})$. The 2mm-diameter Nd:YAG crystal with the diaphragm (the diameter is $1.5 \mathrm{~mm}$ ) on the outlet face was used to obtain the light generation in a single mode regime (single transverse mode). Q-switching was made by the electro-optical shutter, working based on the Pockels effect. Between the Nd:YAG crystal and the shutter, the polarizer, transmitting light only with horizontal polarization, was placed. Thus, at the generator output, we had a single mode beam with horizontal polarization. After the generator, the light beam passed through the linear telescope, which also worked as a rotating prism. The main purpose of this component was to decrease the beam divergence in the horizontal plane, which is necessary for effective conversion of the fundamental frequency to its harmonics. Then, the laser beam was sent to the 90 degree polarization rotator, which changed the horizontal polarization to vertical one (required for reducing energy losses on rotating mirrors). Passed through the rotator and reflected from the rotating mirror beam was directed through the round-trip amplifier, which was composed of the Nd:YAG crystal (5 $\mathrm{mm}$ in diameter) and the rear 
mirror. Then the beam arrived at the polarizer, which reflects only vertical polarization. The reflected beam was directed through the quarter-wave plate for changing the vertical polarization to the circular one. That improves the efficiency of frequency-doubling in a KTP crystal. The KDP and BBO nonlinear crystals were used for generating the $3 \mathrm{~d}$ and 4 th harmonics. After the frequency conversion, the radiation of the 4 th (or the $3 \mathrm{~d}$ ), 2nd and the fundamental harmonics was transmitted through the quartz prism for their spatial divergence in the horizontal plane. The continuous adjustment of the laser intensity during the saturation curves measurement was carried out by the Pockels cell, which was placed in the beam way right after the KTP crystal. The cell consists of the electro-optical component (DKDP crystal) and two Glan prisms. Changing the voltage on the DKDP, one can adjust the radiation output intensity at the outlet from the Pockels cell.

\subsubsection{Registration system}

The laser radiation was focused on the cuvette with the sample solution by a long-focallength lens (the focal length is $20 \mathrm{~cm}$ ). For collecting the fluorescence photons, the lightguide cable (the length is $5 \mathrm{~m}$ ), consisting of seven quartz fibers (the diameter of each fiber is 600 micron), which were laid out in a row (like a slit) at both ends, was used. The cable inlet was fixed at the cuvette side, and the outlet was clasped to the polychromator entrance slit; the sample solution was excited by transmitted laser radiation. As a detector of radiation, the optical multichannel analyser (OMA) was used. The optical chamber (DeltaTech, Scientific Park of MSU) of the analyser consists of the electro-optical converter based on a gated microchannel plate (MCP), CCD matrix, and optical device for transferring an image from the MCP to the CCD matrix (a pixel size is $11 \times 11 \mu \mathrm{m}^{2}$ ). The chamber was fixed to the polychromator (MUM without the output slit, reciprocal linear dispersion is $0.15 \mathrm{~nm}$ per channel) optical output. The multichannel analyser was connected to the PC. As a result, optical image in the polychromator output slit plane could be obtained as a $2 \mathrm{D}$ picture on the PC monitor. The software installed on the PC allowed the OMA to operate both in the continuous and gated modes. When working in gated mode, a part of light was sent to the silica photodiode PD-265 (the building-up time of the leading edge is less than $2 \mathrm{~ns}$ ). The photodiode was connected to the nanogenerator triggering inlet (the trigger level is $0.6 \mathrm{~V}$ ). The gating of the MCP was implemented by high-voltage pulses from the nanogenerator (the amplitude is $800 \mathrm{~V}$ ). The detector gate delay time could be adjusted through the nanogenerator over the range of $50 \mathrm{~ns}$ (the dead time of the detector) to $1200 \mathrm{~ns}$ with the step $t_{\text {step }}=2.5 \mathrm{~ns}$. Exactly because of the dead time of the registration system, when it operates in the gated mode (i.e. in the case of kinetic curves measurements), the light-guide cable as the dead time compensator (the optical delay line) was used. The detector gate width $t_{g}$ could be varied from 10 to 1200 ns; in our experiments it was set to $10 \mathrm{~ns}$.

\subsubsection{Laser radiation parameters}

The laser pulse duration at the wavelengths of 532 and $266 \mathrm{~nm}$ were fitted well by Gaussian function with the full width at half maximum $\left(t_{p}\right)$ of 12 and $10 \mathrm{~ns}$, respectively. When measuring (i) the kinetic curves, the laser beam diameter was $3 \mathrm{~mm}$ and (ii) the saturation curves, the beam was focused to a spot with a diameter from 600 to $800 \mu \mathrm{m}$, depending on the protein under investigation. The values of the photon flux density $F$ were determined according to the equation $F^{-1}=E^{-1} \hbar \omega \cdot S \cdot t_{p}$, where $\hbar \omega$ is a photon energy, $E$ is average pulse 
energy, $S$ is cross section area of a laser beam, $t_{p}$ is a pulse duration. The photon flux density $F_{\max }$ at a maximum of the saturation curve was measured before each experiment. Then, the photon flux density was gradually decreased. The $F$ value at each point was obtained by the value of the Raman scattering signal from water molecules $N_{R S}: F=F_{\max } \cdot N_{R S} / N^{\max }{ }_{R S}$, where $N^{\max } R S$ is the Raman scattering signal at $F=F_{\max }$. In nonlinear laser fluorimetry experiments, the fluorescence saturation curves were measured in the following ranges of $F$ : (i) $5 \times 10^{23} \div 7 \times 10^{25} \mathrm{~s}^{-1} \mathrm{~cm}^{-2}$ when the excitation wavelength was $532 \mathrm{~nm}$ and (ii) $2 \times 10^{24} \div 5 \times 10^{25}$ $\mathrm{s}^{-1} \mathrm{~cm}^{-2}$ when excited at the wavelength of $266 \mathrm{~nm}$.

\subsection{Picosecond time-resolved and steady-state spectral measurements}

The fluorescence lifetime measurements were performed with a streak camera (Agat SF 3M, VNIIOFI, Russia). A Nd:YAG laser with excitation wavelengths of 532 and $266 \mathrm{~nm}$ (the second and fourth harmonic of fundamental radiation) was used as a light source. The laser radiation parameters of the fluorimeter were as follows (for $532 \mathrm{~nm}$ ): pulse energy $160 \mu \mathrm{J}$, duration $20 \mathrm{ps}$ (fwhm), beam diameter $5 \mathrm{~mm}$. The error in determining the fluorescence lifetimes in time intervals of several nanoseconds did not exceed $5 \%$. In addition to the laser equipment, the Cary 100 spectrophotometer (Varian, Inc., USA) and the Cary Eclipse spectrofluorimeter (Varian, Inc., USA; slits width was $5 \mathrm{~nm}$ ) were used for optical density measurements and fluorescence registration, respectively.

\section{The object}

\subsection{Albumins}

In this work, the solutions of human serum albumin (HSA) (>96\%, Sigma) and of bovine serum albumin (BSA) (>98\%, MP Biomedicals) in a phosphate buffer (0.01 M, pH 7.4) have been used. The proteins concentrations were 10-5 (absorption spectra measurement) and 10-9 $\mathrm{M}$ (fluorescence measurement at the nanosecond laser fluorimeter). All of the experiments were performed at a temperature of $25 \pm 1^{\circ} \mathrm{C}$. The structure and biological functions of HSA and BSA can be found in (Peters, 1996). Tryptophan, tyrosine, and phenylalanine (with relative contents of 1:18:31 in HSA and 2:20:27 in BSA) are the absorption groups in these proteins (as in many other natural proteins). The tyrosine fluorescence in HSA and BSA (as in many other natural proteins) is quenched due to the effect of adjacent peptide bonds, polar groups (such as $\mathrm{CO}, \mathrm{NH}_{2}$ ), and other factors, and phenylalanine has a low fluorescence quantum yield (0.03) (Permyakov, 1992). Therefore, the fluorescence signal in these proteins is determined mainly by tryptophan groups. In that case the fluorescence, registered in nonlinear and kinetic laser fluorimetry measurements, correspond to tryptophan residues (this fact will be used in Section 6.1).

As described in (Peters, 1996), HSA and BSA have similar structure and amino acid sequences that differ insignificantly by location of some certain amino acids. However, HSA contains one tryptophan residue in the protein matrix (Trp-214), and BSA contains two residues (Trp-212 and Trp-134). Trp-212 in BSA and Trp-214 in HSA have a similar microenvironment and, hence, their spectral properties are similar (Eftink et al., 1977). Tryptophans of BSA are not spectrally identical due to the stronger integration of Trp-212 into the protein's structure and the more hydrophobic environment of Trp-212 in comparison with Trp-134. The distance between tryptophans in BSA is about $3.5 \mathrm{~nm}$. This 
fact makes the intramolecular energy transfer between them using the Forster resonance energy transfer (FRET (Valeur, 2002)) mechanism possible.

\subsection{Red fluorescent proteins}

Fluorescent proteins are a class of proteins that have a distinguishing property of forming their chromophore without involvement of any additional cofactors and ferments (autocatalytic reaction), except for molecular oxygen. In recent years, FPs have gained enormous popularity as genetically encoded fluorescence markers that enable to visualize a broad range of biological processes in cells and tissues. The most popular for practical applications are FPs whose fluorescence is shifted to the red (red FPs) and whose molecules are monomers (Piatkevich et al, 2010). The mRFP1 protein possesses these properties (Cambel et al., 2002), which made it an object of the research.

In this work the red FP mRFP1 and its mutants at 66 amino acid residue (glutamine 66) have been used. The seven mutants with substitution of the glutamine 66 for the serine (protein mRFP1/Q66S2), cysteine (mRFP1/Q66C), asparagine (mRFP1/Q66N), histidine $(\mathrm{mRFP1} / \mathrm{Q66H})$, alanine (mRFP1/Q66A), leucine (mRFP1/Q66L) and phenylalanine (mRFP1/Q66F) have been created. The method for fabrication and purification of proteins is described in (Vrzheshch et al., 2008). All the experiments were performed in a $0.06 \mathrm{M}$ phosphate buffer at a temperature of $25 \pm 1^{\circ} \mathrm{C}$. The Bradford method (McCluskey, 2003) was used to determine initial concentration of the proteins. The proteins concentration was: (1) $10^{-9} \mathrm{M}$ when measured fluorescence saturation curves; (2) $5.5 \times 10^{-10} \div 3 \times 10^{-10} \mathrm{M}$ (depending on protein) when measured fluorescence quantum yield; (3) $10^{-6} \mathrm{M}$ when measured steadystate spectra and fluorescence life-times on a picoseconds laser fluorimeter.

The formation of a fluorescent molecule of red FPs is a complicated process (usually called maturation) consisting of several stages (Verkhusha et al., 2004; Strack et al., 2010). At some stages, intermediate protein forms are produced, which remain in the resultant specimen (solution) of protein. In other words, the solution of red FPs is an ensemble of protein molecules consisting of several chemically non-equivalent subensembles (a mixture of different spectral forms of FPs). At neutral $\mathrm{pH}$, the solution of mRFP1-like proteins contains three different spectral forms, namely, the mature form (will denote below as $\mathrm{R}$ form), the immature form with protonated chromophore ( $\mathrm{GH}$ form) and the immature form with deprotonated chromophore ( $\mathrm{G}$ form). The detailed information about formation of the chromophore of red FPs and analysis of composition of the samples can be found elsewhere (Verkhusha et al., 2004; Strack et al., 2010). According to a widely used terminology (Verkhusha et al., 2004), I'll also call the mature form as a red form and the immature forms as a green form in this work. The spectral forms can be detected in an absorption spectrum as the bands with maxima in the blue (360-420 nm, GH form), green (450-540 nm, G form) and red (550-600 nm, R form) spectral ranges. It is known (Pakhomov et al., 2004) that the balance between GH and G forms of FP can be disturbed with the changing of the solution acidity or as a result of light (from the UV, blue, or green spectral ranges) influence on the

\footnotetext{
${ }^{2}$ In this work, the name of each mRFP1 mutant is designated like mRFP1/Q66\#, where Q66 is the abbreviation of the glutamine (single letter code is $Q$ ) at position 66, which is substituted. In place of the symbol \#, the single letter code of amino acid the glutamine is substituted for is written down. The list of the amino acids abbreviation can be found elsewhere (Zamyatin, 1972).
} 
protein. Irradiation of the protein molecules by the light from the green spectral range provokes a conversion of $\mathrm{G}$ form molecules to the molecules of $\mathrm{GH}$ form; as a result, the protein solution will represent a mixture of only two forms, namely, GH and $\mathrm{R}$. In current work, for conversion the radiation of an Ar laser (LG-106-M1) at $488 \mathrm{~nm}(300 \mathrm{~mW})$ was used. An increment of the protein solution $\mathrm{pH}$ to 9 leads to a practically total transfer of the $\mathrm{GH}$ form molecules to the $\mathrm{G}$ form molecules (in that case, the protein solution is a mixture of the $\mathrm{G}$ and $\mathrm{R}$ forms). These procedures will be used as a way to decrease the amount of simultaneously existing forms in the mRFP1 solution when the method of the nonlinear fluorimetry will be implemented (see Section 6).

\section{Steady-state fluorescence and absorption spectroscopy}

\subsection{Human and bovine serum albumins}

The absorption spectra of the proteins and (for comparison) the corresponding equimolar solutions (solution of tryptophan, tyrosine, and phenylalanine at the same ratio as they are contained in protein) are shown in Fig. 4. It is seen that the parameters of the absorption bands of proteins do not coincide with the corresponding parameters for the equimolar solutions.
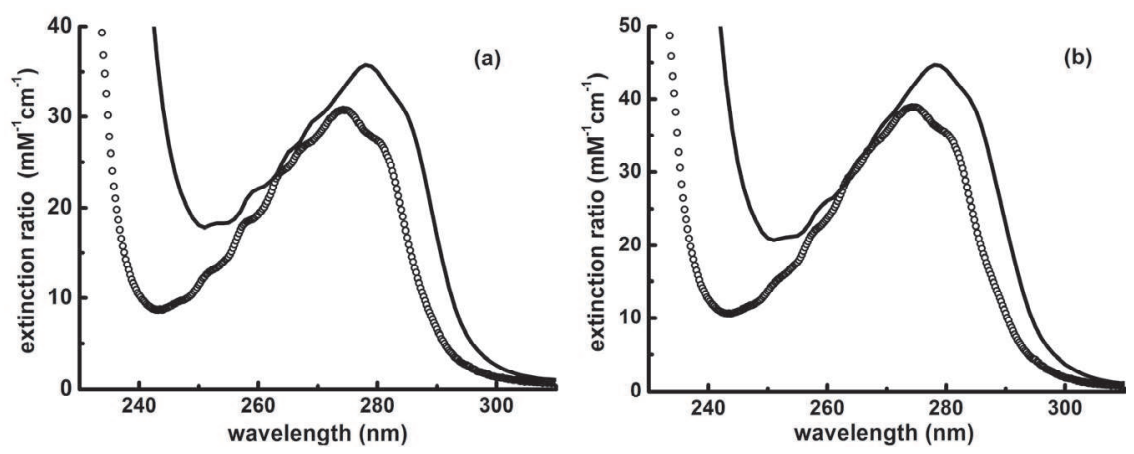

Fig. 4. Solid line is an absorption spectrum of (a) human and (b) bovine serum albumin.

Circles are absorption spectrum of an equimolar solution with relative contents of tryptophan, tyrosine, and phenylalanine as (a) 1:18:31 and (b) 2:20:27.

The measurements of the fluorescence emission spectra of the proteins (data not showed) revealed that the fluorescence of both proteins is blue shifted relative to the tryptophan fluorescence $(353 \mathrm{~nm}$ ) in a buffer solution (Banishev et al., 2008a). This is due to a decrease in the tryptophan environment polarity in the proteins. The maximum of the HSA fluorescence $(332 \mathrm{~nm})$ is blue shifted in comparison with BSA (342 nm). Since the fluorescence spectrum of the tryptophan residues reflects the polarity of their nearest environment, and since the properties of the environments of Trp-212 in BSA and Trp-214 in HSA are similar (Eftink et al., 1977), such a shift can be related to the fact that BSA contains tryptophan Trp-134 located in the environment with a higher polarity (in comparison with Trp-212). Thus, the total fluorescence spectrum of BSA is red shifted. This result will be necessary for choosing the registration wavelength in measuring the acceptor and donor fluorescence when the nonlinear and kinetic curves will be measured (Section 6.1). 


\subsection{Monomeric red fluorescent protein and its mutants at residue 66}

One can see from the absorption spectra of the mRFP1 (Fig. 5(a)) that in the wavelength range from 370 to $650 \mathrm{~nm}$, there exist three absorption bands, which are explained by the presence of three spectral forms in the solution (Verkhusha et al., 2004), i.e. R, G and GH forms (the corresponding absorption maxima at 584, 503 and $380 \mathrm{~nm}$ ). One can also see that the absorption bands of $\mathrm{G}$ and $\mathrm{R}$ forms overlap. Excitation of fluorescence in the absorption band of each form indicates that $\mathrm{G}$ and $\mathrm{GH}$ forms do not fluoresce, the R form fluoresces with maxima at $607 \mathrm{~nm}$. The excitation of the protein solution by irradiation at a wavelength of $270 \mathrm{~nm}$ (tryptophan absorption band) leads to the appearance in the signal spectrum not only of an UV band (maximum at $330 \mathrm{~nm}$ ), which corresponds to the tryptophan fluorescence in the protein matrix, but also of a band in the visible region of wavelengths (maximum at $607 \mathrm{~nm}$ ) corresponding to fluorescence of the chromophore mRFP1 R form (Fig. 5(b)). The chromophore of GH and G forms is non-fluorescent (Campbell et al., 2002).
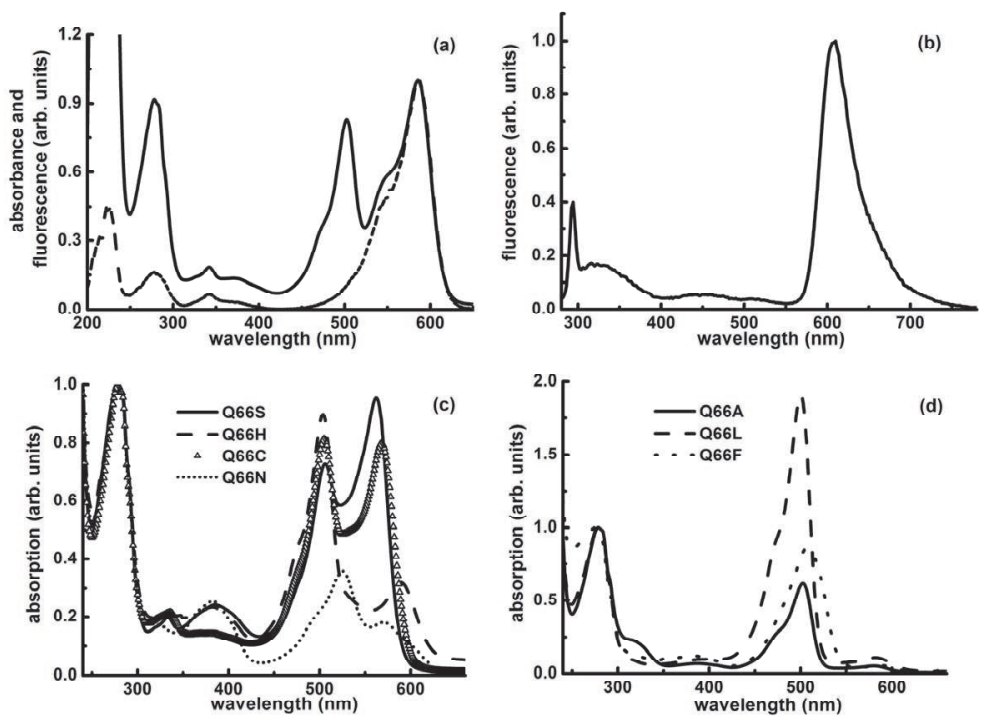

Fig. 5. (a) Absorption (solid) and fluorescence excitation (dotted, registration at $607 \mathrm{~nm}$ ) spectra of mRFP1. The spectra are normalized to the signal at $584 \mathrm{~nm}$. (b) Emission of mRFP1 (excitation by $270 \mathrm{~nm}$ ). (c) Absorption spectra of the mutants with polar and (d) nonpolar substitutions at position 66. The spectra are normalized to optical density at $270 \mathrm{~nm}$.

Absorption spectra of the variants of mRFP1 with mutation at position 66 are shown in the Fig. 5(c, d).The absorption spectra structure of the mutants is similar to one for mRFP1. One can see that for all proteins in the wavelength range from 370 to $650 \mathrm{~nm}$, the spectrum contains three bands with the maxima at 360-420 nm, 450-540 nm and 550-600 nm depending on the protein. These absorption peaks are corresponding to the GH, G and $\mathrm{R}$ forms. There only signal in the red spectral range were observed in the fluorescence emission and excitation spectra (i.e. the $G$ form was found to be non-fluorescent) for all proteins except mRFP1/Q66F. For mRFP1/Q66F, the additional band in fluorescence excitation (maxima at $502 \mathrm{~nm}$ ) and emission (maxima at $512 \mathrm{~nm}$ ) spectra was detected, 
which attributed to the chromophore of $G$ form. The spectral characteristics of the proteins are presented in the Table 1.

Note that the presence of the three protein forms can be qualitatively seen in the absorption spectrum (Fig. 5). However, as is described in (Banishev et al., 2009), the quantitative determination of the individual photophysical parameters of their chromophore with the help of only conventional methods are problematic. This is explained by the fact that the preparative separation of the forms is rather difficult and, as a result, it is hard to find their partial concentrations. At that rate, for example, for calculating the molar extinction coefficient (or absorption cross section) of chromophore from absorption spectra, the total protein concentration (total concentration of all forms) is used. As a result, the extinction coefficients are artificially underestimated (Kredel at al., 2008). At present, the only method that used for determining the individual extinction coefficient of chromophore of each spectral form can be found in (Ward, 2005). However, as it was pointed out in (Kredel et al., 2008), the values measured by the method are inaccurate in case of red FPs. Although, the procedure which enables to reduce the experimental errors has been proposed by (Kredel et al., 2008), the problem remains still topical.

\begin{tabular}{|c|c|c|c|c|c|}
\hline Protein & Residue at 66 & $\begin{array}{c}\text { Green (G) } \\
\text { form }\end{array}$ & \multicolumn{3}{|c|}{ Red form } \\
\cline { 3 - 5 } & & $\lambda_{\text {max }}{ }_{\text {abs }}$ & $\lambda_{\text {max }}{ }_{\text {abs }}$ & $\lambda^{\text {max }_{\text {ex }}}$ & $\lambda_{\text {max }}$ em \\
\hline mRFP1 & Glutamine (Gln) & 503 & 584 & 584 & 607 \\
\hline mRFP1/Q66S & Serine (Ser) & 506 & 562 & 561 & 579 \\
\hline mRFP1/Q66C & Cysteine (Cys) & 505 & 568 & 568 & 588 \\
\hline mRFP1/Q66N & Asparagine (Asn) & 525 & 570 & 570 & 604 \\
\hline mRFP1/Q66H & Histidine (His) & 504 & 588 & 588 & 618 \\
\hline mRFP1/Q66A & Alanine (Ala) & 500 & 582 & 578 & 605 \\
\hline mRFP1/Q66L & Leucine (Leu) & 500 & 582 & 577 & 613 \\
\hline mRFP1/Q66F & Phenylalanine(Phe) & 507 & 591 & 595 & 624 \\
\hline
\end{tabular}

Table 1. The position ( $\mathrm{nm}$ ) of the maximum of the absorption, fluorescence excitation and emission spectra of the proteins.

\section{Nonlinear fluorescence spectroscopy of proteins}

\subsection{Determination of photophysical parameters of single- and double-tryptophan- containing proteins}

The emission spectra at several values of photon flux density $F$ are shown in Fig. 6(a, b). In the figures, the band with a maximum value of $F\left(4 \times 10^{25} \mathrm{~cm}^{-2} \mathrm{~s}^{-1}\right)$ corresponds to the maximum value of the Raman scattering signal from water molecules. One can see that the maximum of the human serum albumin (HSA) fluorescence band does not change its position when $F$ is changed. This is due to the fact that HSA contains one saturating fluorophore. However, the bovine serum albumin (BSA) fluorescence band is blue shifted (from 340 to $335 \mathrm{~nm}$ ) when $F$ is increased, owing to the fact that BSA contains two fluorophores in different environments (therefore, with different spectral properties), which exhibit different degrees (factors) of saturation. Taking into account the blue shift of the HSA fluorescence spectrum (in comparison with the BSA fluorescence spectrum) and the 
similarity of the properties of Trp-214 in HSA and Trp-212 in BSA (see Section 5.1), one can assume that, in the system of two tryptophans of BSA, Trp-212 serves as the donor of the energy (the energy transfer occurs via Forster mechanism), and Trp-214 is the acceptor (i.e., its fluorescence spectrum is presumably shifted towards long wavelengths).
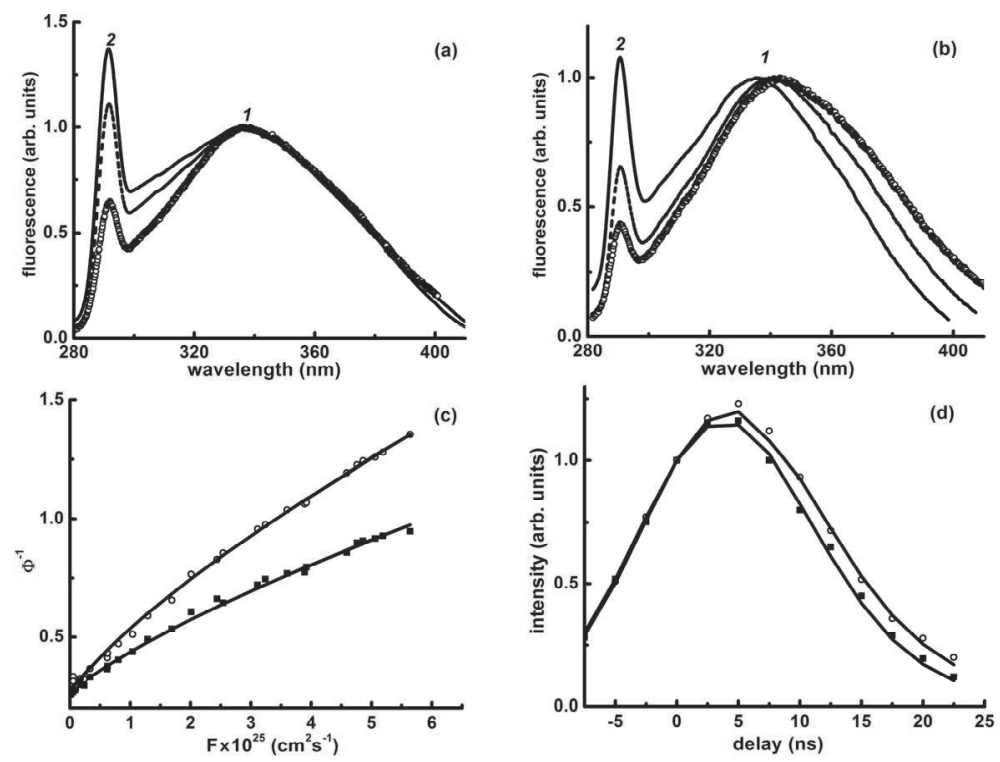

Fig. 6. (a) HSA and (b) BSA emission spectra at several values of photon flux density (see text); (1) is fluorescence and (2) is water Raman scattering band. (c) Saturation and (d) kinetic curves for BSA; fluorescence was registered at 390 (squares) and 310 (circles) nm. Lines are plotted using model $(1 b)$ and Eqs. $(2 b, c)$ for parameters from the Table 2.

The kinetic curves (see Eq. 6) and the fluorescence saturation curves of BSA are shown in Fig. 6(c, d). For BSA, the saturation curves depend on the registration wavelength in the wavelength range $310-390 \mathrm{~nm}$. This is due to the fact that the BSA fluorescence band is a superposition of the bands of two tryptophans possessing different spectral properties. A similar difference in the curves for HSA is negligible. For the determination of the photophysical parameters of HSA fluorophore from fluorescence saturation and kinetic curves, the model (1a) and Eq. (2a) have been used. The same calculation procedure was done for BSA experimental curves, but with using the model (1b) and Eqs. (2b,c); the fluorescence signal was measured at $310 \mathrm{~nm}$ (when registered the fluorescence saturation and kinetic curves of the donor) and $390 \mathrm{~nm}$ (for similar curves of the acceptor). The resulting values of the parameters of protein fluorophores are presented in the Table 2.

As one can see from the Table 2 that the values of photophysical parameters $\sigma$ and $\tau$ of Trp214 in HSA and Trp-212 in BSA are similar. This result should have been expected based on a comparison of the structures of these proteins. The rates of energy transfer in BSA from excited donor to unexcited acceptor $\left(K_{D A}\right)$ and to excited acceptor $\left(K_{S S}\right)$ are small in comparison with the rate of intramolecular relaxation $\left(\tau^{1}\right)$. This can be due to following reasons: (i) in BSA, the tryptophan residues in the D-A pair are located at a distance that is 
insufficient for a noticeable energy transfer between them. According to the data on the BSA structure (Peters, 1996), the distance between two tryptophans in the molecule is about $3.5 \mathrm{~nm}$. For comparison, the Forster radius for the energy transfer between free tryptophans ranges from 0.6 to $1.2 \mathrm{~nm}$ (depending on the solvent). (ii) perhaps, the mutual orientation of the transition dipoles of fluorophores impedes the energy transfer (Lakowicz, 1999).

\begin{tabular}{|c|c|c|c|}
\hline Protein & Parameters & \multicolumn{2}{|c|}{ Tryptophan residues } \\
\hline & & \multicolumn{2}{|c|}{$\operatorname{Tr} p-214$} \\
\hline HSA & $\begin{array}{l}\tau, \mathrm{ns} \\
\sigma\left(\lambda_{\mathrm{ex}}=266\right), \mathrm{cm}^{2} \\
K_{32}^{\prime}, \mathrm{s}^{-1}\end{array}$ & \multicolumn{2}{|c|}{$\begin{array}{c}4.5 \pm 0.5 \\
(1.3 \pm 0.1) \times 10^{-17} \\
<10^{-7}\end{array}$} \\
\hline & & Trp-134 (donor) & Trp-212 (acceptor) \\
\hline BSA & $\begin{array}{l}\tau, \mathrm{ns} \\
\sigma\left(\lambda_{\mathrm{ex}}=266\right), \mathrm{cm}^{2} \\
K_{D A}, \mathrm{~s}^{-1} \\
K_{S S}, \mathrm{~s}^{-1}\end{array}$ & $\begin{array}{c}6.2 \pm 0.5 \\
(3 \pm 0.3) \times 10^{-17} \\
<10^{-7} \\
<10^{-7}\end{array}$ & $\begin{array}{c}5 \pm 0.5 \\
(1 \pm 0.1) \times 10^{-17} \\
<10^{-7} \\
<10^{-7}\end{array}$ \\
\hline
\end{tabular}

Table 2. Photophysical parameters of fluorophores (tryptophan residues) in HSA and BSA.

It is significant that the values of the absorption cross section determined using the method of nonlinear fluorimetry are true values for fluorophores and they are obtained without $a$ priori information about the contribution of other groups into absorption at a specific wavelength and about the concentration of fluorophores. This is a unique feature of nonlinear fluorimetry. As mentioned above, there are three absorption groups in proteins (tryptophan, tyrosine and phenylalanine) and the absorption spectra of these three amino acids are overlapping. In that situation it is hard to separate the contribution of each amino acid group from total protein absorption without any preparative action on a molecule. For that reason, the absorption cross section of the tryptophan residue in protein is assumed to be equal to the absorption cross section of free tryptophan in solution (Pace et al., 1995), because it is supposed that this parameter is weakly dependent on the environment. However, a comparison of the equimolar solution and protein solution absorption spectra (Fig. 4) shows that these spectra do not coincide for HSA and BSA. Therefore, it is clear that such an assumption is just estimation and for diagnostics of the state of a protein it is necessary to be able to determine the true photophysical parameters of tryptophan merged in protein matrix. That has been done in this Section. The absorption cross section for tryptophan in aqueous solution is equal to $1.6 \times 10^{-17} \mathrm{~cm}^{2}$ (Banishev et al., 2008a) (at $266 \mathrm{~nm}$ ); now, it can be compared with the true values of the absorption cross section of tryptophan residues in native proteins (see Table 2). The abilities of the approach are not limited by albumins.

\subsection{Determination of photophysical parameters of mRFP1 protein under UV excitation}

According to three-dimensional structure (PDB ID1g7k) of the mRFP1, there is a tryptophan at a distance of about $15 \AA$ from the protein chromophore, which could be a potential partner (the donor) for inductive FRET to the protein chromophore. Thereby, the tryptophan and the chromophore form a LDA pair inside a molecule of the FP. 
As a preliminary step in determination of the full set of the mRFP1 photopysical parameters, the analysis of fluorescence decay at picoseconds excitation has been done. The excitation wavelength was $266 \mathrm{~nm}$ in order to match the acceptor (tryptophan) absorption band. The acceptor fluorescence decay under excitation of an ensemble of donor-acceptor pairs by $\delta$-pulse is described (Valeur, 2002) as:

$$
\mathrm{I}_{\mathrm{A}}(\mathrm{t}) \sim \mathrm{B} \cdot \exp \left(-\mathrm{t} / \tau_{\mathrm{A}}\right)-\mathrm{A} \cdot \exp \left(-\mathrm{t} / \tau_{\mathrm{D}+\mathrm{A}}\right)
$$

where, $B=A+\left[A^{*}\right]_{0},\left[A^{*}\right]_{0}$ is the excited acceptor molecules concentration at a time point $t=0$ (immediately after the excitation pulse is over); $A=\left[D^{*}\right]_{0} \cdot K_{D A} /\left(1 / \tau_{D+A}-1 / \tau_{A}\right) ;\left[D^{*}\right]_{0}$ is the excited donor molecules concentration at a time point $t=0 ; \tau_{D+A}=\left(1 / \tau_{D}+K_{D A}\right)^{-1}$ is the fluorescence lifetime of the donor in the presence of the acceptor; other designations are given above.

The fluorescence decay signal was measured at picoseconds time-resolved fluorimeter described in Section 3.2. The excitation wavelength was $266 \mathrm{~nm}$ and the signal registration was done in the range of the $\mathrm{R}$ form chromophore fluorescence (the chromophores of others forms are non-fluorescent). The experimental time dependence was fitted by function (4), as a result, the lifetimes $\tau_{A}, \tau_{D+A}$, and the partial contributions of $B$ and $A$ components of the fluorescence decay curve were obtained. The $K_{D A}$ cannot be determined only from the fluorescence decay curve, because in this case it would be necessary to remove the acceptor and measure the fluorescence lifetime of the donor (in the absence of the acceptor). As it will be shown below, the nonlinear fluorimetry method makes it possible to resolve this problem for the native protein without any preparative action. The processing of the fluorescence decay curve of the mRFP1 allowed us to determine the lifetime values, $\tau_{A}=1.6 \mathrm{~ns}$ and $\tau_{D+A}=0.24 \mathrm{~ns}$. The values of $B$ and $A$ in (4) were found to be practically equal. It is indicative of the absence of the direct excitation of the acceptor; therefore, in the equation system (1b) one can assume $\sigma_{A}=0$ (under excitation at $266 \mathrm{~nm}$ ) and for this reason, the acceptor fluorescence (under this wavelength excitation) is a result of the energy transfer from the tryptophan to the chromophore.

The next step was to measure and analyse the fluorescence saturation curves with excitation at the wavelength of $266 \mathrm{~nm}$. To improve the stability of the inverse problem solution, the process of singlet-singlet annihilation from the model (1b) was excluded. At $\mathrm{pH} 7.4$ (the acidity at which the protein was initially produced), the mRFP1 solution is represented as a sum of three subensembles of molecules, each having its own set of photophysical parameters, and the dynamics of the populations of the collective states is described by its own system of equations similar to $(1 \mathrm{~b})$. The number of the photons of the tryptophan fluorescence is calculated from Eq. (2b), where the sum under the integral is the population of the collective states $n_{2}$ and $n_{4}$ for all three forms. The fluorescence of the $\mathrm{R}$ form is calculated from Eq. (2c), where the populations of the collective states $n_{3}$ and $n_{4}$ of this form are present. It is difficult to resolve the inverse problem of nonlinear fluorimetry in such a situation, because the number of unknown parameters is too large. But, as was mentioned in Section 4.2, there are the techniques that allow reducing the number of the simultaneously present forms to two. In that case if the portion of the concentration of the $\mathrm{R}$ form is $c_{R}$ and that of the second form (for example, $G$ ) is $c_{G}$, then the numbers of the fluorescence photons of the donor and acceptor (from the unit of volume) are: 


$$
\begin{gathered}
N_{F l}^{D}(\lambda)=\tau_{D}^{-1} \cdot \eta_{D} \cdot \int_{-\infty}^{+\infty}\left[c_{R} \cdot\left(n_{2}{ }^{R}(t, r)+n_{4}{ }^{R}(t, r)\right)+c_{G} \cdot\left(n_{2}{ }^{G}(t, r)+n_{4}{ }^{G}(t, r)\right)\right] d t \\
N_{F l}^{A}(\lambda)=\tau_{A}^{-1} \cdot \eta_{A} \cdot \int_{-\infty}^{+\infty} c_{R}\left[n_{3}{ }^{R}(t, r)+n_{4}{ }^{R}(t, r)\right] d t
\end{gathered}
$$

where the symbols $\mathrm{R}$ and $\mathrm{G}$ denote the $\mathrm{R}$ and $\mathrm{G}$ form molecules; $n_{2}, n_{3}$, and $n_{4}$ are the collective states (see (1b)); $\eta_{D}$ and $\eta_{A}$ are the fluorescence quantum yields (see $(2 b, c)$ ); and $c$ is the relative concentration of the forms in total molecules concentration. The amount of the $\mathrm{R}$ form molecules $c_{R}$ can be found from the algorithm that described in the next Section.

In this connection, the following procedure has been realized:

a. The value of the protein solution $\mathrm{pH}$ was set near 9; in this situation, the protein solution contains only protein molecules of the $\mathrm{R}$ and $\mathrm{G}$ forms. After that, two saturation curves were taken under excitation at the wavelength of $266 \mathrm{~nm}$ and fluorescence registration at 330nm (the fluorescence saturation curve of the donor, i.e., the molecules of the tryptophan contained in the protein matrix of the R and G forms) and at $607 \mathrm{~nm}$ (the fluorescence saturation curve of the acceptor, i.e., the chromophore only in the protein molecules of the R form). Resolving the inverse problem, in which fluorescence response formation is described by two systems of the kind of $(1 \mathrm{~b})$ for the populations of the collective states of the LDA pairs in macromolecules of $R$ and $G$ forms, and the values of the parameters $\tau_{D+A}, \tau_{A}, \sigma_{A}=0$ for the $R$ form of the protein are considered to be known (see above), the values of $K_{D A}$ (for the $\mathrm{R}$ and $\mathrm{G}$ forms) and $\tau_{A}$ (for the $\mathrm{G}$ form) have been determined.

b. The protein sample was irradiated with the Ar laser at a wavelength of $488 \mathrm{~nm}$ (the $\mathrm{pH}$ value is near to a neutral one) and made only the $\mathrm{R}$ and $\mathrm{GH}$ forms present in the solution. After that, I used the same procedure of measurement and calculation of the saturation curves and determined the following parameters: $K_{D A}$ (for R and GH forms of protein) and $\tau_{A}$ (for the GH form of the protein). The values of the $K_{D A}$ for the $\mathrm{R}$ form in both cases coincided in error limits of the experiment.

The experimental dependences $\Phi^{-1} D(F)$ and $\Phi^{-1} A(F)$ for case (a) can be found in (Shirshin et al., 2009). When measured the saturation curves $\Phi^{-1} D(F)$ and $\Phi^{-1} A(F)$, the intensity in the spectra of the first and second orders of the RS valence band of water molecules (the wavelengths are 291 and $582 \mathrm{~nm}$, correspondingly) were used as a reference signal; the excitation was at $266 \mathrm{~nm}$. Having performed this procedure, the photophysical parameters of FP mRFP1 under UV excitation (266 nm) have been determined (see Table 3).

Let us discuss the main results of this Section. First of all, it is interesting to compare the true value of the absorption cross section obtained for tryptophan in the FP mRFP1 with the values for tryptophan in an aqueous solution $\left(1.6 \times 10^{-17} \mathrm{~cm}^{2}\right.$ (Banishev et al., 2008)), human serum albumin $\left(1.3 \times 10^{-17} \mathrm{~cm}^{2}\right)$, and bovine serum albumin $\left(\sigma_{D}=1 \times 10^{-17} \mathrm{~cm}^{2}\right.$ and $\sigma_{A}=3 \times 10^{-17}$ $\mathrm{cm}^{2}$ ), which were determined in previous Section. One can see that the values for tryptophans in proteins are different and do not equal to the value for a free tryptophan, as is often assumed. I want to emphasize that the lifetime of the excited state of the donor (tryptophan) $\tau_{D}$ in the absence of the acceptor (chromophore) has been obtained without the removal of the acceptor (as it is often supposed when determine the energy transfer 
efficiency in LDA pairs by conventional methods (Valuer, 2002)). For mRFP1, this value can be compared with ones for the free tryptophan $\left(\tau_{D}=2.8 \mathrm{~ns}\right.$ (Banishev et al., 2008a)), HSA ( $\tau_{D}=4.5 \mathrm{~ns}$ ) and BSA ( $\tau_{D}=5$ and $\tau_{A}=6.2 \mathrm{~ns}$ ). Simultaneously, the excited state lifetime values of the chromophores of the $\mathrm{GH}$ and $\mathrm{G}$ form have been obtained ( $\tau_{A}$ in the Table 3$)$, although the chromophores of these forms are non-fluorescent. The obtained results show that the high volume $(E=0.89)$ of the energy transfer efficiency from the tryptophan to the chromophores in all three forms of the protein is of special scientific and practical interest. This permits employing mRFP1 as a promising fluorescence indicator that makes use of its own inner LDA pair (an alternative is the preparation of such pairs of two proteins (Srinivas et al., 2001; Truong \& Ikura, 2001).

\begin{tabular}{|c|c|c|c|}
\hline Parameter & Values for R form & Values for GH form & Values for G form \\
\hline$\sigma_{\mathrm{D}}\left(\lambda_{\mathrm{ex}}=266\right), \mathrm{cm}^{2}$ & $(1 \pm 0.2) \times 10^{-16}$ & $(1 \pm 0.2) \times 10^{-16}$ & $(1 \pm 0.2) \times 10^{-16}$ \\
\hline$\sigma_{\mathrm{A}}\left(\lambda_{\mathrm{ex}}=266\right), \mathrm{cm}^{2}$ & 0 & not defined & not defined \\
\hline $\mathrm{K}_{\mathrm{DA}}, \mathrm{s}^{-1}$ & $(3.7 \pm 0.7) \times 10^{9}$ & $(7.8 \pm 1) \times 10^{9}$ & $(2.5 \pm 0.7) \times 10^{9}$ \\
\hline $\mathrm{E}$ & 0.89 & 0.94 & 0.84 \\
\hline$\tau_{\mathrm{A}}, \mathrm{ns}$ & $3 \pm 0.15$ & $1.9 \pm 0.4$ & $1.7 \pm 0.4$ \\
\hline$\tau_{\mathrm{D}}, \mathrm{ns}$ & $2.1 \pm 0.5$ & $2.1 \pm 0.5$ & $2.1 \pm 0.5$ \\
\hline
\end{tabular}

Table 3. The photophysical parameters of the LDA pairs in mRFP1 by UV excitation. In the table: (1) $\sigma_{D}\left(\lambda_{e x}=266\right)$ and $\sigma_{A}\left(\lambda_{e x}=266\right)$ are the absorption cross section of the donor (tryptophan) and the acceptor (chromophore) at the wavelength of $266 \mathrm{~nm}$; (2) $K_{D A}$ and $E \equiv K_{D A} /\left(K_{D A}+1 / \tau_{D}\right)$ are the rate and efficiency of the energy transfer from the excited donor to the unexcited acceptor; (3) $\tau_{D}$ and $\tau_{A}$ are the excited state lifetimes of the donor (in the absence of the acceptor) and the acceptor.

\subsection{Influence of a single amino-acid substitution on the individual photophysical parameters of the fluorescent form of the mRFP1 protein}

In this Section, the influence of a single amino acid substitution in mRFP1 at position 66 on optical characteristics of the chromophore of fluorescent spectral form ( $R$ form) is performed. For that purpose, the method of nonlinear laser fluorimetry was realized in the version when the protein fluorescence is excited by the wavelength of $532 \mathrm{~nm}$ (i.e. the only protein chromophore was excited). All technical details of the procedure can be found in (Banishev et al., 2009).

At first, the photophysical parameters of $\mathrm{R}$ form chromophore of the proteins were determined. The $\sigma$ and $K_{32}^{\prime}$, defined in Section 2, have been determined from fluorescence saturation curve for each of the eight protein samples. Because the solution of each of the eight proteins contains mature (red) and immature (green) form, the only fluorescence in the red spectral range (from $550 \mathrm{~nm}$ ) was detected (to obtain the parameters only for $\mathrm{R}$ form chromophore). The typical view of the measured fluorescence saturation curves can be found in (Banishev et al., 2009). To simplify the inverse problem solution, the fluorescence lifetime $\tau$ was measured independently with the picosecond laser fluorimeter (excitation at $532 \mathrm{~nm}$ ). It was found that for all protein samples the fluorescence decay best fit by a singleexponential dependence (Banishev et al., 2009). Solving the inverse problem of nonlinear 
fluorimetry for each saturation curve at given $\tau$, the $\sigma$ and $K_{32}^{\prime}$ for each protein sample have been defined. Note that in this scheme of nonlinear laser fluorimetry the 532-nm laser pulses were used for exciting fluorescence, and, hence, $\sigma$ is the absorption cross section of the protein $\mathrm{R}$ form at $532 \mathrm{~nm}$, i.e., $\sigma=\sigma_{R}{ }^{(532)}$.

At the second stage, the partial concentration of the mature and immature species in the resultant solution of each mutant has been determined. As was said above, the equilibrium between the GH and G forms of FPs can be shifted under the action of external factors. Using this property of red FPs, it is possible to find the ratio of concentrations of all forms. However, the only the red fluorescence of $\mathrm{R}$ form is useful for practical application (Piatkevich et al., 2010). The green form is the by-products of maturation and supposed to be absent in ideal case. For that reason the measurement procedure has been simplified and the concentration of the $\mathrm{R}$ form and the total concentration of the $\mathrm{GH}$ and $\mathrm{G}$ forms were determined.

Indeed, given above-mentioned assumptions, one can write the following system of equations:

$$
\begin{aligned}
& \frac{\Phi_{0}{ }^{(570)}}{\Phi_{0}(532)} \cdot \frac{\sigma_{R S}^{(570)}}{\sigma_{R S}^{(532)}}=\frac{\sigma_{R}^{(570)}}{\sigma_{R}^{(532)}} \\
& C_{R} \cdot \sigma_{R}^{(570)}=2.3 D^{(570)} l^{-1} \\
& C_{R} \cdot \sigma_{R}^{(532)}+C_{G} \cdot \sigma_{G}^{(532)}=2.3 D^{(532)} l^{-1} \\
& C_{R}+C_{G}=C_{0},
\end{aligned}
$$

where $C_{R}, C_{G}$ are the concentrations of the $\mathrm{R}$ form and the total concentration of the $\mathrm{G}$ and $\mathrm{GH}$ forms in the solution (in $\mathrm{cm}^{-3}$ ); $C_{0}$ is the total concentration of protein molecules determined by conventional methods (McCluskey, 2003); $\sigma_{R}{ }^{(570)}$ and $\sigma_{R}{ }^{(532)}$ are the individual absorption cross section of the chromophore of fluorescent form at 570 and $532 \mathrm{~nm} ; \sigma_{R}(570)$ is integral absorption cross section of the chromophore of green form; $D^{(570)}, D^{(532)}$ and $\sigma_{R S}(570)$, $\sigma_{R S}{ }^{(532)}$ are the optical density of the protein solution and Raman scattering cross section of water (Filipova et al., 2001) at 570 and $532 \mathrm{~nm}$, respectively.

The first equality in system (6) reflects the fact that the quantum yields (expressed in terms of the fluorescence parameter $\Phi_{0}$ (Filipova et al., 2001)) upon excitation of the protein solution at 532 and $570 \mathrm{~nm}$ are the same. The second and third equalities are the optical density (determined from the absorption spectrum of the proteins) written in terms of the concentration of protein molecules absorbing light at 570 and $532 \mathrm{~nm}$ and in terms of their absorption cross section. The wavelengths of 570 and $532 \mathrm{~nm}$ were chosen for reason mentioned in (Banishev et al., 2009). In system (6) the sought-for quantities are $C_{R}, C_{G}, \sigma_{R}(570)$, $\sigma_{G}{ }^{(532)}$, while experimentally measured values are $\Phi_{0}^{(570)} / \Phi_{0}(532), D^{(570)}, D^{(532)}, l, C_{0}, \sigma_{R}{ }^{(532)}$ (the latter found by means of nonlinear laser fluorimetry). After the $\sigma_{R}{ }^{(570)}$ was found, one can find the maximum value of the individual absorption cross section $\sigma_{R}{ }^{(\max )}$ of the $\mathrm{R}$ form chomophore, or the extinction coefficient $\varepsilon_{R}^{(m a x)}$, which is more convenient for comparison with data from literature. This value can be calculated using the absorption spectrum and relation $D^{(\max )} / D^{(570)}=\varepsilon_{R}(\max ) / \varepsilon_{R}(570)$, where $D^{(\max )}$ is the optical density at the maximum of the absorption band of the $\mathrm{R}$ form. The results for the eight samples are given in the Table 4 . 
One can see from the Table 4 that at the absorption maximum of the R form of the mRFP1 $\varepsilon_{R}{ }^{(\max )}=(215 \pm 40) \mathrm{mM}^{-1} \mathrm{~cm}^{-1}$, which is drastically (four times) larger than the value published in (Campbell et al., 2002). This difference is due to the fact that the (Campbell et al., 2002) calculated the extinction coefficient using the total protein concentration (and, therefore, found the integral extinction coefficient) rather than the partial concentration (as in our case). As a result, the determination of the partial concentration of fluorescent molecules allowed us to find the individual extinction coefficient of the chromophore of the $\mathrm{R}$ form.

\begin{tabular}{|l|c|c|c|c|}
\hline Protein & $\varepsilon_{R}{ }^{(\max )}\left(\mathrm{mM}^{-1} \mathrm{~cm}^{-1}\right)$ & $C_{R} / C_{0}{ }^{* *}, \%$ & $\eta$ & $\eta_{T}{ }^{*}$ \\
\hline mRFP1 & $215 \pm 40$ & $26 \pm 6$ & $0.24 \pm 0.03$ & $0.01 \pm 0.01$ \\
\hline mRFP1/Q66S & $85 \pm 13$ & $34 \pm 6$ & $0.20 \pm 0.04$ & $0.05 \pm 0.02$ \\
\hline mRFP1/Q66C & $135 \pm 20$ & $17 \pm 6$ & $0.19 \pm 0.04$ & $0.01 \pm 0.01$ \\
\hline mRFP1/Q66N & $133 \pm 15$ & $9 \pm 4$ & $0.17 \pm 0.03$ & $0.02 \pm 0.02$ \\
\hline mRFP1/Q66H & $230 \pm 27$ & $8 \pm 4$ & $0.13 \pm 0.03$ & $0.07 \pm 0.02$ \\
\hline mRFP1/Q66A & $171 \pm 16$ & $2 \pm 2$ & $0.19 \pm 0.04$ & $0.05 \pm 0.02$ \\
\hline mRFP1/Q66L & $240 \pm 40$ & $2 \pm 2$ & $0.12 \pm 0.03$ & $0.06 \pm 0.02$ \\
\hline mRFP1/Q66F & $142 \pm 18$ & $2 \pm 2$ & $0.04 \pm 0.03$ & $0.12 \pm 0.03$ \\
\hline
\end{tabular}

Table 4. Individual photophysical parameters of the $\mathrm{R}$ form and its fraction in the protein sample. Note: $\tau, \eta$ and $\eta_{T}$ are the fluorescence lifetime, fluorescence quantum yield and quantum yield to the triplet state (converted from $K_{32}^{\prime}$ ), respectively. Other parameters are defined after the system of Eqs. (6). * Determined from the fluorescence saturation curve; ** determined by solving system of Eqs. (6).

In the general case, the determination of photophysical parameters of FPs with the help of integral characteristics of the sample is incorrect, which can be proved by several examples. By using the dynamic difference method, (Kredel et al., 2008) obtained the individual extinction coefficient $143 \mathrm{mM}^{-1} \mathrm{~cm}^{-1}$ for the chromophore of the $\mathrm{R}$ form of $\mathrm{mPlum}$, which is also drastically larger than other published values ranging from 22 to $41 \mathrm{mM}^{-1} \mathrm{~cm}^{-1}$ (Shcherbo et al., 2007) for this protein. It is interesting to note that (Gross et al., 2000) have earlier reported a similar value of $150 \mathrm{mM}^{-1} \mathrm{~cm}^{-1}$ for the $\mathrm{R}$ form chromophore of red FP DsRed (the table value for this protein is assumed to be $75 \mathrm{mM}^{-1} \mathrm{~cm}^{-1}$ ). In their approach, the amount of immature species was deduced from mass spectroscopic analysis. Another example can be found in (Strack et al., 2010). Using the method described in (Ward, 2005), (Strack et al., 2010) got an assessed value of $123 \mathrm{mM}^{-1} \mathrm{~cm}^{-1}$ for DsRed.T7, which is close to that obtained by (Kredel et al., 2008). From these examples one can see that the values of the individual extinction coefficients of the $\mathrm{R}$ form chromophore are close, as it is expected to be, because the chromophores of these proteins are considered to be chemically identical. One can assume a minor disagreement due to chromophore orientation change relative to protein matrix or composition of its closest environment (this can explain the difference in the extinction values for mPlum, DsRed and DsRed.T7 determined by (Kredel et al., 2008; Gross et al., 2000) and (Strack et al., 2010)). However, the published values of the extinction of red FPs with the same chromophore drastically vary depending on the protein: 75 $\mathrm{mM}^{-1} \mathrm{~cm}^{-1}$ per a polypeptide chain for the DsRed, $120 \mathrm{mM}^{-1} \mathrm{~cm}^{-1}$ per a polypeptide chain for tdimer2(12) (Campbell at al., 2002), $22 \mathrm{mM}^{-1} \mathrm{~cm}^{-1}$ for mPlum (Shcherbo et al., 2007), $50 \mathrm{mM}$ ${ }^{1} \mathrm{~cm}^{-1}$ for mRFP1 (Campbell at al., 2002) and $90 \mathrm{mM}^{-1} \mathrm{~cm}^{-1}$ for mStrawberry (Shu et al., 2006). Discrepancies follow directly from the content of immature form in the protein samples. 
In the measurements I obtained $215 \mathrm{mM}^{-1} \mathrm{~cm}^{-1}$ for mRFP1, which is larger than the value for DsRed and mPlum. However, it should be noted that I did not take into account the photochemical processes (photoionisation, photobleaching, etc. (Banishev et al., 2008a) in the model of fluorescence response generation (1a). The efficiency of these processes in the protein samples under study may be different. When efficient enough, the photochemical processes may contribute noticeably to fluorescence saturation. In this case their emission can result in the saturation curve giving an overstated value of the absorption cross section and, therefore, overstated quantity of $\varepsilon_{R}{ }^{(\max )}$. On the other hand, as it was mentioned in Section 5.2, the method applied by (Kredel et al., 2008) for determining the individual extinction coefficient of mPlum is not accurate enough in the case of red FPs (the method is well adapted only for GFP-like FPs). Therefore, the obtained value of $143 \mathrm{mM}^{-1} \mathrm{~cm}^{-1}$ can be underestimated and the precise value of the chromophore extinction is greater.

As it was shown earlier (Banishev et al., 2009), for the $R$ form of the proteins mRFP1, $\mathrm{mRFP1/Q66S} \mathrm{and} \mathrm{mRFP1/Q66C,} \mathrm{the} \mathrm{position} \mathrm{of} \mathrm{the} \mathrm{maximum} \mathrm{of} \mathrm{absorption,} \mathrm{fluorescence}$ excitation and emission bands depends on the substituted amino-acid residue at position 66 and positively correlates with the volume of this residue: the maximum moves to the red range with increasing the volume of the residue. A similar correlation was described for the individual extinction coefficient of the $\mathrm{R}$ form chromophore, i.e. a higher extinction coefficient corresponds to a larger volume of the residue. The results for the new mutants mRFP1/Q66N, mRFP1/Q66H, mRFP1/Q66A, mRFP1/Q66L and mRFP1/Q66F are presented in Fig. 7(a, b). In the same figure the results obtained in (Banishev et al., 2009) for mRFP1, mRFP1/Q66S and mRFP1/Q66C are plotted. The values of the amino acids volume were taken from (Zamyatin, 1972). One can see that the dependence of the position of steady-state spectra (at maximum) of two new mutants (mRFP1/Q66N and mRFP1/Q66H) on the volume of amino-acid residue at position 66 has the same trend as described in (Banishev et al., 2009). The same can be observed for the individual extinction coefficient (but not for the integral one). There are no such dependences for characteristics of the proteins mRFP1/Q66A, mRFP1/Q66L and mRFP1/Q66F.

The results can be explained in the following way. It is known that formation of $\mathrm{R}$ form chromophore of red FPs goes through formation of a double bond between the Ca and $\mathrm{N}$ atoms of the 66th amino acid residue. Since dehydrogenation of a bond between Ca and N atoms involves the carbonaceous framework of the 66th amino acid residue in the system of conjugation, then the changes in the side radical of this residue can lead to the changes in the spectral and photophysical properties of the new mutants.

In the case of a polar amino acid at position 66 (serine, cysteine, asparagines and histidine), its side radical can form hydrogen bonds with the side radicals of glutamine-42, glutamine213 and glutamate-215 (see Fig. 7 (c, d)). These radicals, in turn, belong to the protein shell (the $\beta$-barrel) and are rather rigidly bonded to it (Khrameeva et al., 2008). A change in the geometry of the side radical at position 66 will in this case cause a change in the geometry of the chromophore imidazolidine ring, because the interaction of the chromophore with the glutamine- 42 and glutamine-213 through the hydrogen bond network can be distorted and a new bond with glutamate- 215 can be formed. As a result, the chromophore tilt- and twistangles (the pictures with the explanation of the angles can be found in (Piatkevich et al., 2010)) will change and the chromophore coplanarity will be distorted. In (Piatkevich et al., 2010) it was shown on the basis on x-ray diffraction data that the chromophore planarity is 
directly connected with the optical properties (the steady-state spectra positions, fluorescence quantum yield, etc.) of red FPs. The deviations from chromophore coplanarity are responsible for the changes in the optical characteristics for mCherry and mStrawberry (Shu et al., 2006). The interrelation between the optical properties of the monomeric red FPs and the geometry of their chromophores was also confirmed by the molecular dynamics simulations conducted for mRFP1 mutants with single polar amino acid substitutions at position 66 (Khrameeva et al., 2008). The simulations have shown that the substitutions have an influence on the torsion angles in the phenolic and imidazolidine rings of the chromophore as well as on the torsion angles in the regions of connection between these rings and chromophore attachment to $\beta$-barrel. It was predicted that the volume of the amino acid residue at position 66 can correlate with the optical characteristics of the mutants. The experimental results presented in this Section are consistent with the results of simulations performed by (Khrameeva et al., 2008).
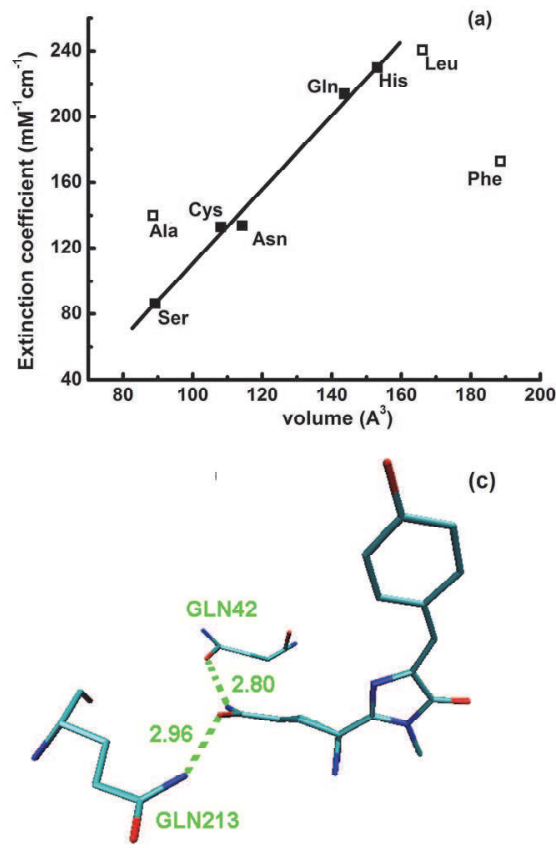

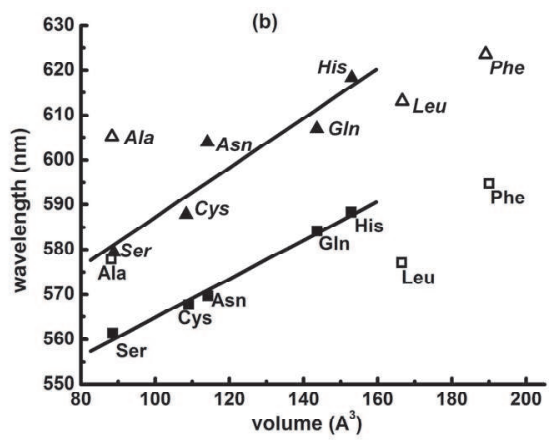

(d)

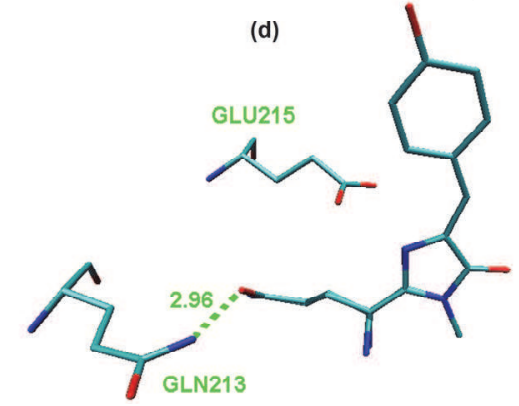

Fig. 7. The dependence of the (a) extinction coefficient, (b) position of absorption/ excitation (squares) and fluorescence emission (triangles) maximum of the protein $\mathrm{R}$ form on the volume of 66th amino acid residue. The solid and hollow scatters are polar and non-polar amino acids, respectively. (c) and (d) are schematic diagrams of chrmophore environment, showing the residues location at positions 213, 42 and 213, 215, respectively. Hydrogen bonds are shown in dashed lines, labeled with lengths in angstroms. Glu is glutamate.

For the mutants with non-polar groups at residue 66 (alanine, leucine and phenylalanine) there is no correlation effect. The non-polar substitutions lead to breakage of hydrogen bonds between the 66th residue of the chromophore and glutamine- 42 and glutamine- 213 . Formation of a new bond between the chromophore and the glutamate-215 is unlikely 
because of non-polarity of substituted residues. Therefore, there is no defined correlation between the chromophore geometry (consequently, the volume of the substituted amino acid residue) and the optical properties of the proteins.

At the present time the red FPs whose molecules are monomers are of particular interest (Piatkevich et al., 2010) as fluorescent markers. Attempts to find new variants of red FPs in order to improve their properties (higher brightness, photo and $\mathrm{pH}$ stability, etc.) are performed. However, the interrelation between optical or photophysical parameters and structural properties of FPs, which is necessary for development of these studies, is rather unclear. A method for prediction of properties of FPs based on their structure is still not developed. This problem might be solved by analysis of properties of mutant proteins with point mutations. Therefore, the results obtained in this Section can be used to tackle the general problem of the development of an algorithm, which could provide the prediction of the spectral properties of FPs based on their structures. The data will also be useful for revealing promising positions for directed mutagenesis.

\section{Conclusion}

In current work the approach based on the simultaneous use of nonlinear laser fluorimetry, spectrophotometry and conventional fluorimetry methods has been applied for investigation of the photophysical properties of the protein molecules of different complexity. The full set of photophysical parameters of the fluorophores (tryptophan residues) of human and bovine serum albumins has been determined. The photophysical processes in the spectral forms of the red FP mRFP1 under UV $(266 \mathrm{~nm})$ and visible $(532 \mathrm{~nm})$ irradiation are described quantitatively. The individual photophysical parameters of the new mutants of the mRFP1 protein (a single substitution at the 66 amino acid position) were determined. It was shown that the individual extinction coefficient of the red chromopore of the proteins correlate positively with the volume of the substituted amino acid residue at position 66 (for polar substitution). A similar correlation has been described for the position of the maximum of the absorption, fluorescence excitation and emission spectra: the position of the maximum moves to the red with increasing the volume of the residue. In addition, the partial concentration of the fluorescent spectral form in the resultant solution of each FP variant has been determined.

\section{Acknowledgment}

The author is grateful to Prof. Victor Fadeev for providing the ability to work in his group and for great help in mastering the fluorescence spectroscopy methods. The author also thanks Evgeny Vrzheshch for the samples of the red FPs and for valuable discussions.

\section{References}

Agranovich, V.M. \& Galanin, M.D. (1982). Electronic Excitation Energy Transfer in Condensed Matter, Elsevier Science Ltd, ISBN 978-0444863355, North-Holland

Banishev, A.A.; Vrzhechsh, E.P. \& Shirshin, E.A. (2009). Application of laser fluorimetry for determining the influence of a single amino-acid substitution on the individual 
photophysical parameters of a fluorescent form of a fluorescent protein mRFP1. IEEE J. Quantum Electron., Vol. 39, No. 3, pp. 273-278, ISSN 10637818

aBanishev, A.A.; Shirshin, E.A. \& Fadeev, V.V. (2008). Determination of photophysical parameters of tryptophan molecules by methods of laser fluorimetry. IEEE J. Quantum Electron, Vol. 38, No. 1, pp. 77-81, ISSN 10637818

bBanishev, A.A.; Shirshin, E.A. \& Fadeev, V.V. (2008). Laser fluorimetry of proteins containing one and two tryptophan residues. Laser Physics, Vol. 18, No. 7, pp. 861867, ISSN 1054-660X

Banishev, A.A.; Maslov, D.V. \& Fadeev, V.V. (2006). A nanosecond laser fluorimeter. Instrum. Exp. Tech., Vol. 49, No. 3, pp. 430-434, ISSN 0020-4412

Boychuk, I.V.; Dolenko, T.A.; Sabirov, A.R.; Fadeev, V.V.; Filippova, E.M. (2000). Study of the uniqueness and stability of the solutions of inverse problem in saturation fluorimetry. IEEE J. Quantum Electron, Vol. 30 , No. 7, pp. 611-616, ISSN 10637818

Campbell, R.E.; Tour, O.; Palmer, A.E.; Steinbach, P.A.; Baird, G.S., Zacharias, D.A. \& Tsien, R.Y. (2002). A monomeric red fluorescent protein. Proc. Natl. Acad. Sci. USA, Vol. 99, pp. 7877-7882, ISSN 1091-6490

Eftink, M.R.; Zajicek, J.L. \& Ghiron, C.A. (1977). A hydrophobic quencher of protein fluorescence: 2,2,2-trichloroethanol. Biochim Biophys Acta., Vol. 491, No. 2, pp. 473481, ISSN 0006-3002

Fadeev, V.V.; Dolenko, T.A.; Filippova, E.M. \& Chubarov, V.V. (1999). Saturation spectroscopy as a method for determining the photophysical parameters of complicated organic compounds. Opt. Commun., Vol. 166, pp. 25-33, ISSN 0030-4018

Filipova, E.M.; Fadeev, V.F.; Chubarov, V.V.; Dolenko, T.A.\& Glushkov, S.M. (2001). Laser fluorescence spectroscopy as a method for determining humic substance. Appl. Spectrosc., Vol. 36, No. 1, pp. 87-117, ISSN 0003-7028

Gross, L.A.; Baird, G.S.; Hoffman, R.C.; Baldridge, K.K. \& Tsien, R.Y. (2000). The structure of the chromophore within DsRed, a red fluorescent protein from coral. Proc. Natl. Acad. Sci. USA, Vol. 97, pp. 11990-11995, ISSN 1091-6490

Khrameeva, E.E.; Drutsa, V.L.; Vrzheshch, E.P.; Dmitrienko, D.V. \& Vrzheshch, P.V. (2008). Mutants of monomeric red fluorescent protein mRFP1 at residue 66: structure modeling by molecular dynamics and search for correlations with spectral properties. Biochemistry, Vol. 73, No. 10, pp. 1082-1095, ISSN 0006-2979

Kredel, S.; Nienhaus, K.; Oswald, F.; Wolff, M.; Ivanchenko, S.; Cymer, F.; Jeromin, A.; Michel, F. J.; Spindler, K.D.; Heilker, R.; Nienhaus, G.U \& Wiedenmann, J. (2008). Optimized and far-red-emitting variants of fluorescent protein eqFP611. Chem. Biol., Vol. 15, pp. 224-233, ISSN 1074-5521

Lakowicz, Joseph R. (1999). Principles of Fluorescence Spectroscopy (2nd edition), Kluwer/Plenum Publishers, ISBN 0-306-46093-9, New York

McCluskey, K. (2003). The fungal genetics stock center: from molds to molecules. Adv. Appl. Microbiol., Vol. 52, pp. 245-262, ISSN 1365-2672

Pace, C.N.; Vajdos, F. \& Fee, L. (1995). How to measure and predict the molar absorption coefficient of a protein. Protein Sci., Vol. 4, pp. 2411, ISSN 1469-896x

Pakhomov, A.A.; Martynova, N.Y.; Gurskaya, N.G.; Balashova, T.A. \& Martynov, V.I. (2004). Photoconversion of the chromophore of a fluorescent protein from Dendronephthya sp. Biochemistry, Vol. 69, pp. 901-908, ISSN 0006-2979 
Permyakov, E.A. (1992). Luminescent Spectroscopy of Proteins, CRC Press Inc, ISBN 9780849345531, Boca Raton, USA

Peters, Jr., T. (1996). All About Albumin: Biochemistry, Genetics, and Medical Applications, Academic Press, ISBN 978-0125521109, San Diego, USA

Piatkevich, K.D.; Efremenko, E.N.; Verkhusha, V.V. \& Varfolomeev S.D. (2010). Red fluorescent proteins and their properties. Russ. Chem. Rev., Vol. 79, No. 3, pp. 243258, ISSN 1468-4837

Shcherbo, D.; Merzlyak, E.M.; Chepurnykh, T.V.; Fradkov, A.F.; Ermakova, G.V.; Solovieva, E.A.; Lukyanov, K.A.; Bogdanova, E.A.; Zaraisky, A.G.; Lukyanov, S. \& Chudakov, D.M. (2007). Bright far-red fluorescent protein for whole-body imaging. Nat. Methods, Vol. 4, pp. 741-746, ISSN 1548-7091

Shirshin, E.A.; Banishev, A.A. \& Fadeev, V.V. (2009). Localized donor-acceptor pairs of fluorophores: determination of the energy transfer rate by nonlinear fluorimetry. JETP Letters, Vol. 89, No. 10, pp. 475-478, ISSN 0021-3640

Shu, X.; Shaner, N.C.; Yarbrough, C.A.; Tsien, R.Y. \& Remington, S.J. (2006). Novel chromophores and buried charges control color in mFruits. Biochemistry, Vol. 45, No. 32, pp. 9639-47, ISSN 1520-4995

Srinivas, G.; Yethiraj, A. \& Bagchi, B. (2001). FRET by FET and dynamics of polymer folding. J. Phys. Chem. B, Vol. 105, pp. 2475-2478, ISSN 1520-5207

Strack, L.R.; Strongin, D.E.; Benjamin, L.M.; Glick, S. \& Keenan, R.J. (2010). Chromophore formation in DsRed occurs by a branched pathway. J. Am. Chem. Soc., Vol. 132, pp. 8496-8505, ISSN 0002-7863

Truong, K. \& Ikura, M. (2001). The use of FRET imaging microscopy to detect proteinprotein interactions and protein conformational changes in vivo. Curr. Opin. Struct. Biol. Vol. 11, pp. 573-578, ISSN 0959-440X

Valeur, B. (2002). Molecular Fluorescence: Principles and Applications, Wiley-VCH, ISBN 9783527299195, Weinheim, Germany

Verkhusha, V.V.; Chudakov, D. M.; Gurskaya, N. G.; Lukyanov, S.; Lukyanov K.A. (2004) Common pathway for the red chromophore formation in fluorescent proteins and chromoproteins. Chem. Biol., Vol. 11, pp. 845-854, ISSN 1074-5521

Vrzheshch, E.P.; Dmitrienko, D.V.; Rudanov, G. S.; Zagidullin, V.E.; Paschenko, V. Z.; Razzhivin, A.P.; Saletsky, A.M. \& Vrzheshch, P. V. (2006). Optical properties of the monomeric red fluorescent protein mRFP1. Moscow Univ. Phys. Bull., Vol. 63, No. 3, pp. 109-112, ISSN 0096-3925

Ward, W.W. (2005). Biochemical and Physical Properties of Green Fluorescent Protein. In Green Fluorescent Protein: Properties, Applications and Protocols (2nd Edition), Wiley John \& Sons , ISBN 9780471736820, New Jersey, USA

Zamyatin, A.A. (1972). Protein Volume in Solution. Prog. Biophys. Mol. Biol., Vol. 24, pp. 107123, ISSN 0079-6107.

Protein structure available from Protein Data Bank, www.pdb.org, ID 2vad 


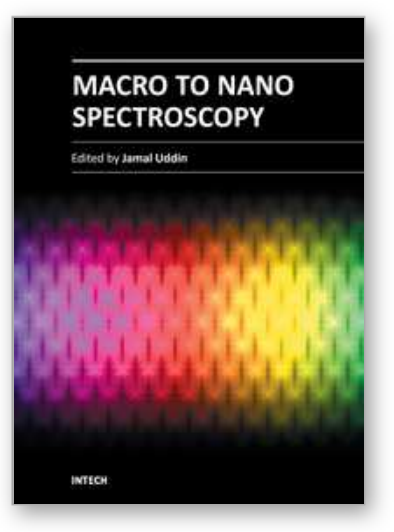

\author{
Macro To Nano Spectroscopy \\ Edited by Dr. Jamal Uddin
}

ISBN 978-953-51-0664-7

Hard cover, 448 pages

Publisher InTech

Published online 29, June, 2012

Published in print edition June, 2012

In the last few decades, Spectroscopy and its application dramatically diverted science in the direction of brand new era. This book reports on recent progress in spectroscopic technologies, theory and applications of advanced spectroscopy. In this book, we (INTECH publisher, editor and authors) have invested a lot of effort to include 20 most advanced spectroscopy chapters. We would like to invite all spectroscopy scientists to read and share the knowledge and contents of this book. The textbook is written by international scientists with expertise in Chemistry, Biochemistry, Physics, Biology and Nanotechnology many of which are active in research. We hope that the textbook will enhance the knowledge of scientists in the complexities of some spectroscopic approaches; it will stimulate both professionals and students to dedicate part of their future research in understanding relevant mechanisms and applications of chemistry, physics and material sciences.

\title{
How to reference
}

In order to correctly reference this scholarly work, feel free to copy and paste the following:

Alexander A. Banishev (2012). Laser Fluorescence Spectroscopy: Application in Determining the Individual Photophysical Parameters of Proteins, Macro To Nano Spectroscopy, Dr. Jamal Uddin (Ed.), ISBN: 978-95351-0664-7, InTech, Available from: http://www.intechopen.com/books/macro-to-nano-spectroscopy/laserfluorescence-spectroscopy-application-in-determining-the-individual-photophysical-paramete

\section{INTECH}

open science | open minds

\author{
InTech Europe \\ University Campus STeP Ri \\ Slavka Krautzeka 83/A \\ 51000 Rijeka, Croatia \\ Phone: +385 (51) 770447 \\ Fax: +385 (51) 686166 \\ www.intechopen.com
}

\author{
InTech China \\ Unit 405, Office Block, Hotel Equatorial Shanghai \\ No.65, Yan An Road (West), Shanghai, 200040, China \\ 中国上海市延安西路65号上海国际贵都大饭店办公楼 405 单元 \\ Phone: +86-21-62489820 \\ Fax: +86-21-62489821
}


(C) 2012 The Author(s). Licensee IntechOpen. This is an open access article distributed under the terms of the Creative Commons Attribution 3.0 License, which permits unrestricted use, distribution, and reproduction in any medium, provided the original work is properly cited. 